\title{
On Training Targets for Supervised LPC Estimation to Augmented Kalman Filter-based Speech Enhancement
}

This paper was downloaded from TechRxiv (https://www.techrxiv.org).

\section{LICENSE}

CC BY 4.0

SUBMISSION DATE / POSTED DATE

22-04-2021 / 30-04-2021

\section{CITATION}

Roy, Sujan Kumar; Nicolson, Aaron; Paliwal, Kuldip K. (2021): On Training Targets for Supervised LPC Estimation to Augmented Kalman Filter-based Speech Enhancement. TechRxiv. Preprint. https://doi.org/10.36227/techrxiv.14463435.v1

$\mathrm{DOI}$ 


\title{
On Training Targets for Supervised LPC Estimation to Augmented Kalman Filter-based Speech Enhancement
}

\author{
Sujan Kumar Roy ${ }^{\mathrm{a}, *}$, Aaron Nicolson $^{\mathrm{b}}$, Kuldip K. Paliwal ${ }^{\mathrm{a}}$ \\ ${ }^{a}$ Signal Processing Laboratory, Griffith University, Nathan Campus, Brisbane, QLD, 4111, Australia \\ ${ }^{b}$ Australian eHealth Research Centre, CSIRO, Herston, QLD, 4006, Australia
}

\begin{abstract}
The performance of speech coding, speech recognition, and speech enhancement largely depends upon the accuracy of the linear prediction coefficient (LPC) of clean speech and noise in practice. Formulation of speech and noise LPC estimation as a supervised learning problem has shown considerable promise. In its simplest form, a supervised technique, typically a deep neural network (DNN) is trained to learn a mapping from noisy speech features to clean speech and noise LPCs. Training targets for DNN to clean speech and noise LPC estimation fall into four categories: line spectrum frequency (LSF), LPC power spectrum (LPC-PS), power spectrum (PS), and magnitude spectrum (MS). The choice of appropriate training target as well as the DNN method can have a significant impact on LPC estimation in practice. Motivated by this, we perform a comprehensive study on the training targets using two state-of-the-art DNN methods- residual network and temporal convolutional network (ResNet-TCN) and multi-head attention network (MHANet). This study aims to determine which training target as well as DNN method produces more accurate LPCs in practice. We train the ResNet-TCN and MHANet for each training target with a large data set. Experiments on the NOIZEUS corpus demonstrate that the LPC-PS training target with MHANet produces a lower spectral distortion (SD) level in the estimated speech LPCs in real-life noise conditions. We also construct the AKF with the estimated speech and noise LPC parameters from each training target using ResNet-TCN and MHANet. Subjective AB listening tests and seven different objective quality and intelligibility evaluation measures (CSIG, CBAK, COVL, PESQ, STOI, SegSNR, and SI-SDR) on the NOIZEUS corpus demonstrate that the AKF constructed with MHANet-LPC-PS driven speech and noise LPC parameters produced enhanced speech with higher quality and intelligibility than competing methods.
\end{abstract}

Keywords: Speech enhancement, augmented Kalman filter, LPC, residual network, temporal convolutional network, multi-head attention network.

\section{Introduction}

Speech processing applications, such as low-bit rate audio coding, speech enhancement, speech recognition, rely upon the accuracy of linear prediction coefficient (LPC) estimates of clean speech and noise in practice (Vaseghi, 2006, Chapter 8). For example, the inaccurate estimates of speech and noise LPC parameters impact the quality and intelligibility of enhanced speech produced by an augmented Kalman filter (AKF) (Gibson et al., 1991). To address this, deep learning assisted LPC estimation has been integrated with Kalman filter (KF) and AKF for speech enhancement. For instance, a DNN assisted LPC estimation (DNN-LPC) (Pickersgill et al., 2018), a fully-connected feed-forward DNN (denoted as FNN) assisted KF (FNN-KF) (Yu et al., 2019), FNN and LSTMbased LPC estimation for the colored KF (FNN-CKFS and

\footnotetext{
${ }^{*}$ Corresponding author

Email addresses: sujankumar.roy@griffithuni.edu.au (Sujan Kumar Roy), aaron.nicolson@csiro.au (Aaron Nicolson), k.paliwal@griffith.edu.au (Kuldip K. Paliwal)
}

LSTM-CKFS) (Yu et al., 2020), a deep learning framework (denoted as Deep Xi (Nicolson and Paliwal, 2019)) and whitening filter assisted KF (Deep Xi-KF) (Roy et al., 2020a) and AKF (Deep Xi-AKF) (Roy et al., 2020b) have been introduced. This paper focuses on training targets for supervised LPC estimation with an application of AKFbased speech enhancement.

Paliwal and Basu (1987) introduced the KF for speech enhancement in white noise condition. For the KF, each clean speech frame is represented by an auto-regressive (AR) process, whose parameters comprise the LPC and prediction error variance. The LPC parameters and additive noise variance are used to construct the KF recursive equations. Given a frame of noisy speech samples, the KF gives a linear MMSE estimate of the clean speech samples using the recursive equations. Paliwal and Basu (1987) demonstrated that the inaccurate estimates of the LPC parameters and noise variance result in poor quality and intelligibility in the enhanced speech produced by the KF. Gibson et al. (1991) introduced an AKF for speech enhancement in coloured noise conditions. In AKF, both the clean speech and additive noise are represented by two 
AR processes. The speech and noise LPC parameters form an augmented matrix, which is used to construct the recursive equations of the AKF. In this speech enhancement algorithm (SEA), the AKF processes the noisy speech iteratively (usually three-four iterations) to eliminate the colored background noise, yielding the enhanced speech. During this, the LPC parameters for the current frame are computed from the corresponding filtered speech frame of the previous iteration (Gibson et al., 1991). Although the iterative AKF improves the signal-to-noise ratio (SNR) of noisy speech, the resultant enhanced speech suffers from musical noise and speech distortion. Therefore, the AKF method (Gibson et al., 1991) does not adequately address the LPC parameter estimates in practice.

George et al. (2018) proposed a robustness metric-based tuning of the AKF for enhancing colored noise corrupted speech (denoted as AKF-RMBT). They demonstrated that the inaccurate estimates of the clean speech and noise LPC parameters introduce bias in the $\mathrm{AKF}$ gain, leading to a degradation in speech enhancement performance. To address this, the noise LPC parameters are computed from the first noisy speech frame by assuming that there remains no speech. The computed noise LPC parameters remain constant during processing all noisy speech frames for a given utterance. A whitening filter is also constructed with the constant noise LPCs to pre-whiten each noisy speech frame prior to computing the speech LPC parameters. As a result, the speech and noise LPC parameter estimation process in AKF-RMBT do not adequately address the noise conditions that have time-varying amplitudes. Moreover, the adjusted AKF gain is under-estimated in speech regions, resulting in distorted speech. Roy and Paliwal (2020) proposed an extension of the AKF-RMBT (George et al., 2018) by employing a sensitivity metricbased tuning of the AKF (denoted as AKF-SMBT). In AKF-SMBT, the speech and noise LPC parameters are computed with a similar process as in AKF-RMBT (George et al., 2018). It is demonstrated that the application of sensitivity metric in AKF-SMBT minimizes the underestimation issue of AKF gain, particularly in speech regions as compared to AKF-RMBT. It is also shown that the reduced-biased AKF gain in AKF-SMBT minimizes the amount of residual noise and distortion in the enhanced speech as compared to AKR-RMBT. However, AKFSMBT also does not adequately address speech enhancement for conditions that have time-varying amplitudes.

Deep learning has been used for LPC estimation-a key parameter for KF and AKF-based SEA (Gibson et al., 1991; Paliwal and Basu, 1987). Pickersgill et al. (2018) proposed a deep learning-based LPC estimation method (DNN-LPC). In DNN-LPC, a traditional DNN (originally proposed in (Xu et al., 2014)) learns a mapping from each frame of the noisy speech log power spectra (LPS) to the log LPC power spectra of clean speech. Then the estimated log LPC power spectra is converted to LPC power spectra (LPC-PS) followed by an inverse Fourier transform of it gives the autocorrelation matrix. By solving the au- tocorrelation matrix using the Levinson-Durbin recursion (Vaseghi, 2006, Chapter 8), yielding the LPC parameters of the clean speech. The spectral distortion (SD) (dB) measure has been used to evaluate the performance of the estimated LPCs. However, the SD evaluation results were not given for low SNR levels (below $10 \mathrm{~dB}$ ). Moreover, only six noise recordings were used for training the DNN, reducing its generalisation capabilities for unseen noise conditions.

$\mathrm{Yu}$ et al. (2019) proposed a deep learning assisted KF for speech enhancement (FNN-KF). A traditional FNN (containing input layer, three hidden layers, and output layer) is used as deep learning method. The line spectrum frequency (LSF) (Itakura, 1975) of clean speech (12 order) is used as a training target for the FNN. Specifically, the FNN learns a mapping from each frame of the noisy speech LSFs to the clean speech LSFs. During inference, the estimated LFSs are converted to clean speech LPCs. For training the FNN, only 10720 samples constructed from 670 speech recordings, including four noise recordings and four SNR levels was used. The small training data reduces the generalizing capabilities of the FNN to unseen conditions. In addition, the additive noise variance is computed from the first noisy speech frame by assuming that there remains no-speech. However, this does not account for conditions that have time-varying amplitudes. Later on, Yu et al. (2020) used FNN and LSTM networks to estimate the speech and noise LPCs for colored KF-based speech enhancement (FNN-CKFS and LSTM-CKFS). Specifically, the authors used a similar FNN as in (Yu et al., 2019), while the LSTM network is constructed by stacking input layer, two LSTM layers (with 512 units in each layer), one feed-forward layer with 512 units, and an output layer. The FNN and LSTM learn a mapping from each frame of the noisy speech LSFs to the clean speech and noise LSFs. Then convert the estimated LSFs to the clean speech and noise LPCs. Besides, a maximum likelihood (ML) approach (Srinivasan et al., 2006) is employed to estimate the prediction error variances of the speech and noise AR processes. However, FNN-CKFS and LSTM-CKFS do not adequately address speech and noise LPC parameter estimates in various noise condition-leading to the use of multi-band spectral subtraction (MB-SS) (Kamath and Loizou, 2002) for post-processing. This could be due to training the FNN and LSTM with a small dataset (Yu et al., 2019).

Roy et al. (2020a) incorporated the DeepMMSE framework (Zhang et al., 2020) with KF (denoted as Deep XiKF, since DeepMMSE uses Deep Xi (Nicolson and Paliwal, 2019)) for speech enhancement. In Deep Xi-KF, the DeepMMSE framework (Zhang et al., 2020) within a residual network and temporal convolutional network (ResNetTCN) (Bai et al., 2018; He et al., 2016) has been used to estimate noise power spectral density (PSD) for each noisy speech frame. Roy et al. (2020b) proposed a deep learningbased AKF for speech enhancement (Deep Xi-AKF). In this method, a ResNet-TCN within the Deep Xi frame- 
work (Nicolson and Paliwal, 2019); Deep Xi-ResNet-TCN is used to estimate the noise PSD for each noisy speech frame. Roy and Paliwal (2020a) proposed a causal convolutional encoder-decoder (CCED)-based AKF for speech enhancement (denoted as CCED-AKF). In this method, the CCED maps each frame of the noisy speech magnitude spectrum (MS) to the noise magnitude spectrum, from where the noise PSD is computed. Roy and Paliwal (2020b) proposed a deep residual network assisted AKF for speech enhancement (denoted as ResNet-AKF). Different from MS or PSD estimation of noise, the ResNet learns a mapping from the noisy speech waveform to the noise waveform. In Deep Xi-KF, Deep Xi-AKF, CCEDAKF, and ResNet-AKF (Roy et al., 2020a,b; Roy and Paliwal, 2020a,b), the noise parameters are computed from the estimated noise. A whitening filter is also constructed with its coefficients computed from the estimated noise to pre-whiten each noisy speech frame prior to computing the speech LPC parameters. As demonstrated in (George et al., 2018), the whitening filter partially reduces bias in the speech LPC parameters. Thus, Deep Xi-KF, Deep XiAKF, CCED-AKF, and ResNet-AKF (Roy et al., 2020a,b; Roy and Paliwal, 2020a,b) do not adequately address the speech LPC parameter estimates in practice.

Recently, a DeepLPC framework (Roy et al., 2021a) within ResNet-TCN is proposed to jointly estimate the speech and noise LPC power spectra (LPC-PS). The clean speech and noise LPC parameters are computed from the LPC-PS estimates. The DeepLPC-ResNet-TCN demonstrates a lower SD level in the estimated speech LPCs than the competing methods. This leads to the production of the highest quality and most intelligible enhanced speech amongst current KF and AKF SEAs - outperforming Deep $\mathrm{Xi}-\mathrm{KF}$ while using the same training set. However, the multi-head attention network (MHANet) has been shown to outperform ResNet-TCN for speech enhancement. Motivated by this, Roy et al. (2021b) proposed an extension of the DeepLPC framework (Roy et al., 2021a) within MHANet, called DeepLPC-MHANet, to further improving the speech and noise LPC parameter estimates for the AKF. The DeepLPC-MHANet demonstrates a lower SD level in the estimated speech LPCs than DeepLPCResNet-TCN (Roy et al., 2021a) in various noise conditions. In addition, the AKF constructed with the DeepLPCMHANet driven speech and noise LPC parameters produces higher quality and intelligible enhanced speech than DeepLPC-ResNet-TCN-AKF (Roy et al., 2021a).

Motivated by the recent improvement on deep learning assisted LPC estimation, this paper aims to perform a comprehensive study on LSF, LPC-PS, power spectrum (PS), and magnitude spectrum (MS) training targets using two state-of-the-art deep learning methods - ResNetTCN and MHANet. The aim of this study is to determine which training target as well as deep learning method produces more accurate speech and noise LPC parameters in real-life noise conditions. Due to this purpose, we train the ResNet-TCN and MHANet for each training target using a large data set as found in (Roy et al., 2021b). We compare the SD level of the estimated speech LPCs for each training target and the deep learning method. We also verify the performance of estimated LPCs in speech enhancement context, where AKF is constructed with the estimated speech and noise LPCs from different training targets using MHANet and ResNet-TCN. Specifically, we evaluate the performance of enhanced speech produced by AKF using subjective AB listening tests and seven different objective quality and intelligibility measures (CSIG, CBAK, COVL, PESQ, STOI, SegSNR, and SI-SDR). The SD level evaluation as well as the subjective and objective experiments are conducted on the NOIZEUS corpus in the presence of real-world non-stationary and colored noise conditions for a wide range of SNR levels.

The structure of this paper is as follows: background knowledge is presented in Section 2, including the signal model and the AKF for speech enhancement. In Section 3 , we present the supervised LPC estimation framework, including the training targets. Following this, Section 4 describes the details of DNN methods used in evaluating the training targets. After that, Section 5 describes the experimental setup in terms of speech corpus and objective and subjective evaluation measures. The experimental results are then presented in Section 6. Finally, Section 7 gives some concluding remarks.

\section{Background}

\subsection{Signal model}

The noisy speech $y(n)$, at discrete-time sample $n$, is assumed to be given by:

$$
y(n)=s(n)+v(n),
$$

where $s(n)$ is the clean speech and $v(n)$ is uncorrelated additive coloured noise. Since the LPC estimation as well as AKF-based speech enhancement operates on a frameby-frame basis, firstly, a $32 \mathrm{~ms}$ rectangular window with $50 \%$ overlap is used to convert $y(n)$ into frames, denoted by $y(n, l)$ :

$$
y(n, l)=s(n, l)+v(n, l),
$$

where $l \epsilon\{0,1, \ldots, L-1\}$ is the frame index, $L$ is the total number of frames in an utterance, and $N$ is the total number of samples within each frame, i.e., $n \epsilon\{0,1, \ldots, N-1\}$.

\subsection{AKF for speech enhancement}

For simplicity, the frame index is omitted in the AKF recursive equations. Each frame of the clean speech and noise signal in (2) can be represented with $p^{\text {th }}$ and $q^{\text {th }}$ order AR models, as in (Vaseghi, 2006, Chapter 8):

$$
\begin{aligned}
& s(n)=-\sum_{i=1}^{p} a_{i} s(n-i)+w(n), \\
& v(n)=-\sum_{k=1}^{q} b_{k} v(n-k)+u(n),
\end{aligned}
$$


where $\left\{a_{i} ; i=1,2, \ldots, p\right\}$ and $\left\{b_{k} ; k=1,2, \ldots, q\right\}$ are the LPCs. $w(n)$ and $u(n)$ are assumed to be white noise with zero mean and variances $\sigma_{w}^{2}$ and $\sigma_{u}^{2}$, respectively.

Equations (2)-(4) can be used to form the following augmented state-space model (ASSM) of the AKF, as in (Gibson et al., 1991):

$$
\begin{aligned}
& \boldsymbol{x}(n)=\boldsymbol{\Phi} \boldsymbol{x}(n-1)+\boldsymbol{r} \boldsymbol{g}(n), \\
& y(n)=\boldsymbol{c}^{\top} \boldsymbol{x}(n) .
\end{aligned}
$$

In the above ASSM,

1. $\boldsymbol{x}(n)=\left[\begin{array}{lll}s(n) \ldots s(n-p+1) v(n) \ldots v(n-q+1) & \ldots\end{array}\right]^{T}$ is a $(p+q) \times 1$ state-vector,

2. $\boldsymbol{\Phi}=\left[\begin{array}{cc}\boldsymbol{\Phi}_{s} & 0 \\ 0 & \boldsymbol{\Phi}_{v}\end{array}\right]$ is a $(p+q) \times(p+q)$ state-transition matrix with:

$$
\begin{gathered}
\boldsymbol{\Phi}_{s}=\left[\begin{array}{ccccc}
-a_{1} & -a_{2} & \ldots & -a_{p-1} & -a_{p} \\
1 & 0 & \ldots & 0 & 0 \\
0 & 1 & \ldots & 0 & 0 \\
\vdots & \vdots & \ddots & \vdots & \vdots \\
0 & 0 & \ldots & 1 & 0
\end{array}\right], \\
\boldsymbol{\Phi}_{v}=\left[\begin{array}{ccccc}
-b_{1} & -b_{2} & \ldots & -b_{q-1} & -b_{q} \\
1 & 0 & \ldots & 0 & 0 \\
0 & 1 & \ldots & 0 & 0 \\
\vdots & \vdots & \ddots & \vdots & \vdots \\
0 & 0 & \ldots & 1 & 0
\end{array}\right],
\end{gathered}
$$

3. $\boldsymbol{r}=\left[\begin{array}{cc}\boldsymbol{r}_{s} & 0 \\ 0 & \boldsymbol{r}_{v}\end{array}\right]$, where $\boldsymbol{r}_{s}=\left[\begin{array}{llll}1 & 0 & \ldots & 0\end{array}\right]^{\top}, \boldsymbol{r}_{v}=$ $\left[\begin{array}{llll}1 & 0 & \ldots & 0\end{array}\right]^{\top}$,

4.

$$
\boldsymbol{g}(n)=\left[\begin{array}{l}
w(n) \\
u(n)
\end{array}\right]
$$

5. $\boldsymbol{c}^{\top}=\left[\begin{array}{ll}\boldsymbol{c}_{s}^{\top} & \boldsymbol{c}_{v}^{\top}\end{array}\right]$, where $\boldsymbol{c}_{s}=\left[\begin{array}{llll}1 & 0 & \ldots & 0\end{array}\right]^{\top}$ and $\boldsymbol{c}_{v}=\left[\begin{array}{llll}1 & 0 & \ldots & 0\end{array}\right]^{\top}$ are $p \times 1$ and $q \times 1$ vectors,

6. $y(n)$ is the noisy measurement at sample $n$.

For each frame, the AKF computes an unbiased linear MMSE estimate, $\hat{\boldsymbol{x}}(n \mid n)$ at sample $n$, given $y(n)$, by using the following recursive equations (Gibson et al., 1991):

$$
\begin{aligned}
& \hat{\boldsymbol{x}}(n \mid n-1)=\boldsymbol{\Phi} \hat{\boldsymbol{x}}(n-1 \mid n-1) \\
& \boldsymbol{\Psi}(n \mid n-1)=\boldsymbol{\Phi} \boldsymbol{\Psi}(n-1 \mid n-1) \boldsymbol{\Phi}^{\top}+\boldsymbol{Q r r}^{\top} \\
& \boldsymbol{K}(n)=\boldsymbol{\Psi}(n \mid n-1) \boldsymbol{c}\left(\boldsymbol{c}^{\top} \boldsymbol{\Psi}(n \mid n-1) \boldsymbol{c}\right)^{-1} \\
& \hat{\boldsymbol{x}}(n \mid n)=\hat{\boldsymbol{x}}(n \mid n-1)+\boldsymbol{K}(n)\left[y(n)-\boldsymbol{c}^{\top} \hat{\boldsymbol{x}}(n \mid n-1)\right] \\
& \boldsymbol{\Psi}(n \mid n)=\left[\boldsymbol{I}-\boldsymbol{K}(n) \boldsymbol{c}^{\top}\right] \boldsymbol{\Psi}(n \mid n-1)
\end{aligned}
$$

where $\boldsymbol{Q}=\left[\begin{array}{cc}\sigma_{w}^{2} & 0 \\ 0 & \sigma_{u}^{2}\end{array}\right]$ is the process noise covariance.

For a noisy speech frame, the error covariances $(\boldsymbol{\Psi}(n \mid n-$ 1 ) and $\boldsymbol{\Psi}(n \mid n)$ corresponding to $\hat{\boldsymbol{x}}(n \mid n-1)$ and $\hat{\boldsymbol{x}}(n \mid n))$ and the Kalman gain $\boldsymbol{K}(n)$ are continually updated on a sample-by-sample basis, while $\left(\left\{a_{i}\right\}, \sigma_{w}^{2}\right)$ and $\left(\left\{b_{k}\right\}, \sigma_{u}^{2}\right)$ remain constant. At sample $n, \boldsymbol{h}^{\top} \hat{\boldsymbol{x}}(n \mid n)$ gives the output of the AKF, $\hat{s}(n \mid n)$, where $\boldsymbol{h}=\left[\begin{array}{lllll}1 & 0 & 0 & \ldots & 0\end{array}\right]^{\top}$ is a $(p+q) \times 1$ column vector. As demonstrated in AKF-RMBT (George et al., 2018), $\hat{s}(n \mid n)$ is given by:

$$
\begin{gathered}
\hat{s}(n \mid n)=\left[1-K_{0}(n)\right] \hat{s}(n \mid n-1)+K_{0}(n)[y(n)- \\
\hat{v}(n \mid n-1)],
\end{gathered}
$$

where $K_{0}(n)$ is the $1^{\text {st }}$ component of $\boldsymbol{K}(n)$, given by (George et al., 2018):

$$
K_{0}(n)=\frac{\alpha^{2}(n)+\sigma_{w}^{2}}{\alpha^{2}(n)+\sigma_{w}^{2}+\beta^{2}(n)+\sigma_{u}^{2}},
$$

where

$$
\alpha^{2}(n)=\boldsymbol{c}_{s}^{\top} \boldsymbol{\Phi}_{s} \boldsymbol{\Psi}_{s}(n-1 \mid n-1) \boldsymbol{\Phi}_{s}^{\top} \boldsymbol{c}_{s}
$$

and

$$
\beta^{2}(n)=\boldsymbol{c}_{v}^{\top} \boldsymbol{\Phi}_{v} \boldsymbol{\Psi}_{v}(n-1 \mid n-1) \boldsymbol{\Phi}_{v}^{\top} \boldsymbol{c}_{v}
$$

are the transmission of a posteriori error variances of the speech and the noise augmented dynamic model from the previous sample, $n-1$, respectively (George et al., 2018).

Equation (15) reveals that $K_{0}(n)$ has a significant impact on $\hat{s}(n \mid n)$. In practice, the inaccurate estimates of $\left(\left\{a_{i}\right\}, \sigma_{w}^{2}\right)$ and $\left(\left\{b_{k}\right\}, \sigma_{u}^{2}\right)$ introduce bias into $K_{0}(n)$, which impacts $\hat{s}(n \mid n)$. In this paper, we investigate both the appropriate training target as well as a deep learning method for more accurate estimates of $\left(\left\{a_{i}\right\}, \sigma_{w}^{2}\right)$ and $\left(\left\{b_{k}\right\}, \sigma_{u}^{2}\right)$, leading to an improved $\hat{s}(n \mid n)$.

\section{Supervised LPC estimation}

The supervised LPC estimation framework is shown in Fig. 1. It can be seen that the framework is fed as input the single-sided noisy speech magnitude spectrum $\left|\boldsymbol{Y}_{l}\right|=$ $\{|Y(l, 0)|,|Y(l, 1)|, \ldots,|Y(l, M-1)|\}$. This is computed from the noisy speech in Equation 1 using the short-time Fourier transform (STFT):

$$
Y(l, m)=S(l, m)+V(l, m),
$$

where $Y(l, m), S(l, m)$, and $V(l, m)$ denote the complexvalued STFT coefficients of the noisy speech, clean speech, and noise, respectively, for time-frame index $l$ and discretefrequency bin $m$. The Hamming window is used for analysis and synthesis. In this framework, a DNN learns to map from $\left|\boldsymbol{Y}_{l}\right|$ to the speech and noise features (in concatenated form) $\hat{\boldsymbol{\zeta}}_{l}$. In this study, LSF, LPC-PS, PS, and MS (Sections 3.1-3.4) of the speech and noise are used as 


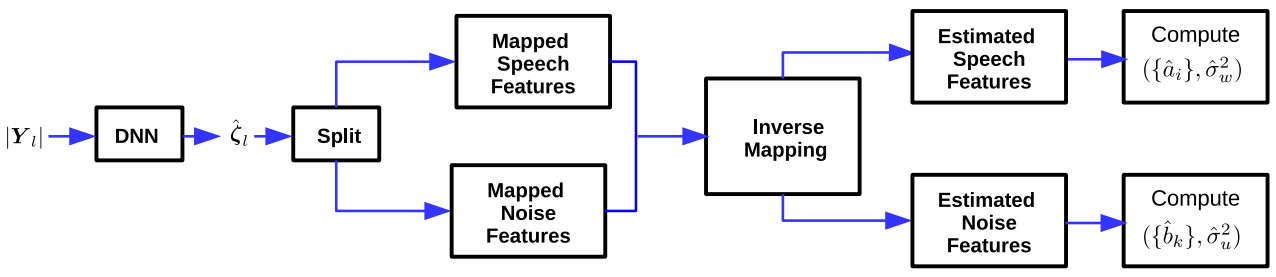

Figure 1: (Color online) Supervised LPC estimation framework.

training targets for the DNN. In addition, ResNet-TCN and MHANet are used as DNN methods. During inference, split $\hat{\boldsymbol{\zeta}}_{l}$ into mapped features of speech and noise followed by inverse mapping of them, yielding the estimated features for speech and noise. Then compute $\left(\left\{\hat{a}_{i}\right\}\right.$, $\left.\hat{\sigma}_{w}^{2}\right)$ and $\left(\left\{\hat{b}_{k}\right\}, \hat{\sigma}_{u}^{2}\right)$ from the estimated speech and noise features. As in DeepLPC-ResNet-TCN framework (Roy et al., 2021a), we have used the speech and noise LPC order; $p=16$ and $q=16$, respectively.

\subsection{LSF training target}

The LSFs of speech and noise (denoted as $\left\{\rho_{i}\right\}$ and $\left.\left\{\eta_{i}\right\}\right)$ are used as training targets for DNN in LPC estimation framework (Fig. 1). During training the DNN, the clean speech and noise are known (the oracle case). Therefore, we first compute the speech and noise LPC parameters, $\left(\left\{a_{i}\right\}, \sigma_{w}^{2}\right)(p=16)$ and $\left(\left\{b_{k}\right\}, \sigma_{u}^{2}\right)(q=16)$ from $s(n, l)$ and $v(n, l)$ using the autocorrelation method as in (Vaseghi, 2006, Chapter 8). Then compute $\left\{\rho_{i}\right\}$ and $\left\{\eta_{k}\right\}$ from $\left\{a_{i}\right\}$ and $\left\{b_{k}\right\}$. We briefly demonstrate the LPC to LSF conversion processes for clean speech as in (McLoughlin, 2008). Each $s(n, l)$ under the linear prediction analysis model can be generated as the output of finite impulse response filter, $A(z)$. $\left\{a_{i}\right\}$ computed from $s(n, l)$ are used to generate $A(z)$ as (McLoughlin, 2008):

$$
A(z)=1+a_{1} z^{-1}+a_{2} z^{-2}+\cdots+a_{p} z^{-p} .
$$

To compute LSFs, $A(z)$ is decomposed into symmetrical and anti-symmetrical parts, represented by the polynomials, $P(z)$ and $Q(z)$, as McLoughlin (2008):

$$
\begin{aligned}
& P(z)=A(z)+z^{-(p+1)} A\left(z^{-1}\right), \\
& Q(z)=A(z)-z^{-(p+1)} A\left(z^{-1}\right) .
\end{aligned}
$$

$\left\{\rho_{i}\right\}$ are expressed as the zeros (or complex roots denoted by $\left.\left\{\theta_{i}\right\}\right)$ of $P(z)$ and $Q(z)$ in terms of angular frequency. Then $\left\{\rho_{i}\right\}$ are computed as (McLoughlin, 2008):

$$
\left\{\rho_{i}\right\}=\tan ^{-1}\left(\frac{\operatorname{Re}\left\{\theta_{i}\right\}}{\operatorname{Im}\left\{\theta_{i}\right\}}\right), i=1,2, \ldots, p,
$$

where $\left\{\rho_{i}\right\}$ are expressed in radians (between $[0, \pi]$ ).

Using eq. (20)-(23), LSFs for the noise signal, $\left\{\eta_{k}\right\}$ are computed from $\left\{b_{k}\right\}$. To improve the rate of convergence of stochastic gradient descent algorithm, the dynamic range of $\left\{\rho_{i}\right\}$ and $\left\{\eta_{k}\right\}$ are compressed to the interval $[0,1]$ as: $\overline{\boldsymbol{\rho}}_{l}=\left\{\frac{\rho_{1}}{\pi}, \frac{\rho_{2}}{\pi}, \ldots, \frac{\rho_{p}}{\pi}\right\}$ and $\overline{\boldsymbol{\eta}}_{l}=\left\{\frac{\eta_{1}}{\pi}, \frac{\eta_{2}}{\pi}, \ldots, \frac{\eta_{q}}{\pi}\right\}$.
The traditional FNN and LSTM in (Yu et al., 2020) learn a mapping form noisy speech LSFs to the clean speech and noise LSFs. Then the estimated LSFs are converted to $\left(\left\{\hat{a}_{i}\right\}\right.$ and $\left\{\hat{b}_{k}\right\}$. However, the prediction error variances, $\hat{\sigma}_{w}^{2}$ and $\hat{\sigma}_{u}^{2}$ are estimated using a ML approach (Srinivasan et al., 2006) with estimated $\left\{\hat{a}_{i}\right\}$ and $\left\{\hat{b}_{k}\right\}$. In this study, we jointly estimate $\left(\left\{\hat{a}_{i}\right\}, \hat{\sigma}_{w}^{2}\right)$ and $\left(\left\{\hat{b}_{k}\right\}, \hat{\sigma}_{u}^{2}\right)$ using the LPC estimation framework (Fig. 1). Due to this purpose, for $l^{\text {th }}$ frame, we concatenate $\overline{\boldsymbol{\rho}}_{l}, \overline{\boldsymbol{\eta}}_{l}, \sigma_{w}^{2}$, and $\sigma_{u}^{2}$ to form $\boldsymbol{\zeta}_{l}$ of size 34 as:

$$
\boldsymbol{\zeta}_{l}=\left\{\bar{\rho}_{1}, \bar{\rho}_{2}, \ldots, \bar{\rho}_{p}, \bar{\eta}_{1}, \bar{\eta}_{2}, \ldots, \bar{\eta}_{q}, \sigma_{w}^{2}, \sigma_{u}^{2}\right\} .
$$

Then $\zeta_{l}$ is used as the final training target for the DNN in the supervised LPC estimation framework (Fig. 1). During inference, $\hat{\boldsymbol{\zeta}}_{l}$ is split into $\hat{\overline{\boldsymbol{\rho}}}_{l}, \hat{\overline{\boldsymbol{\eta}}}_{l}, \hat{\sigma}_{w}^{2}$, and $\hat{\sigma}_{u}^{2}$. Then multiply $\hat{\overline{\boldsymbol{\rho}}}_{l}, \hat{\overline{\boldsymbol{\eta}}}_{l}$ by $\pi$ (inverse mapping), yields $\hat{\boldsymbol{\rho}}_{l}$ and $\hat{\boldsymbol{\eta}}_{l}$. Finally, convert $\hat{\boldsymbol{\rho}}_{l}$ and $\hat{\boldsymbol{\eta}}_{l}$ into $\left\{\hat{a}_{i}\right\}$ and $\left\{\hat{b}_{k}\right\}$ using LSF to LPC conversion method as in (McLoughlin, 2008).

\section{2. $L P C-P S$ training target}

The LPC-PS of speech and noise signal, $P_{s}(l, m)$ and $P_{v}(l, m)$ were used as the training targets in DeepLPCResNet-TCN and DeepLPC-MHANet (Roy et al., 2021a,b). During training, $P_{s}(l, m)$ and $P_{v}(l, m)$ are computed as in (Vaseghi, 2006, Chapter 9):

$$
\begin{aligned}
& P_{s}(l, m)=\frac{\sigma_{w}^{2}}{\left|1+\sum_{i=1}^{p} a_{i} e^{-j 2 \pi i m / M}\right|^{2}}, \\
& P_{v}(l, m)=\frac{\sigma_{u}^{2}}{\left|1+\sum_{k=1}^{q} b_{k} e^{-j 2 \pi k m / M}\right|^{2}},
\end{aligned}
$$

where $m \epsilon\{0,1, \ldots, M-1\}(M=257)$.

As demonstrated in DeepLPC-ResNet-TCN (Roy et al., 2021a), the dynamic range of $P_{s}(l, m)$ and $P_{v}(l, m)$ are compressed to the interval $[0,1]$ through utilizing the cumulative distribution function (CDF) of $P_{s}(l, m)_{[\mathrm{dB}]}$ and $P_{v}(l, m)_{[\mathrm{dB}]}$, where $P_{s}(l, m)_{[\mathrm{dB}]}=10 \log _{10}\left(P_{s}(l, m)\right)$ and $P_{v}(l, m)_{[\mathrm{dB}]}=10 \log _{10}\left(P_{v}(l, m)\right)$. For example, Figs. $2(\mathrm{a})$ and $(\mathrm{c})$ show that $P_{s}(l, 64)_{[\mathrm{dB}]}$ and $P_{v}(l, 64)_{[\mathrm{dB}]}$ follow a Gaussian distribution. In light of the analysis, we assume that $P_{s}(l, m)_{[\mathrm{dB}]}$ and $P_{v}(l, m)_{[\mathrm{dB}]}$ are distributed normally with mean, $\mu_{s}$ and $\mu_{v}$, and variance $\sigma_{s}^{2}$ and $\sigma_{v}^{2}$, respectively $\left(P_{s}(l, m)_{[\mathrm{dB}]} \sim \mathcal{N}\left(\mu_{s}, \sigma_{s}^{2}\right)\right.$ and $\left.P_{v}(l, m)_{[\mathrm{dB}]} \sim \mathcal{N}\left(\mu_{v}, \sigma_{v}^{2}\right)\right)$. The statistics of $P_{s}(l, m)_{[\mathrm{dB}]}$ and $P_{v}(l, m)_{[\mathrm{dB}]}$, i.e., $\left(\mu_{s}, \sigma_{s}^{2}\right)$ 


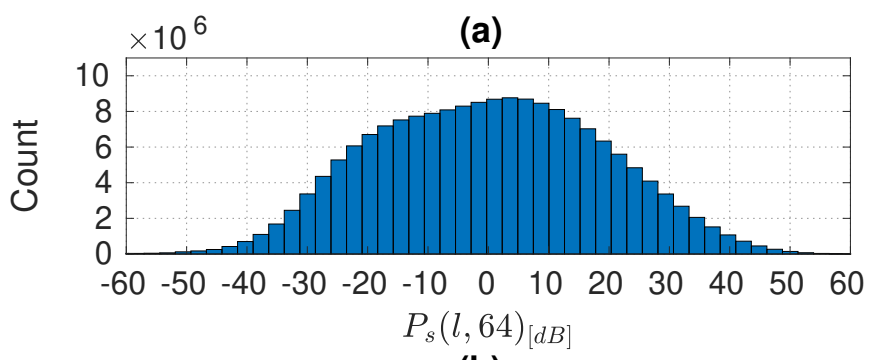

(b)

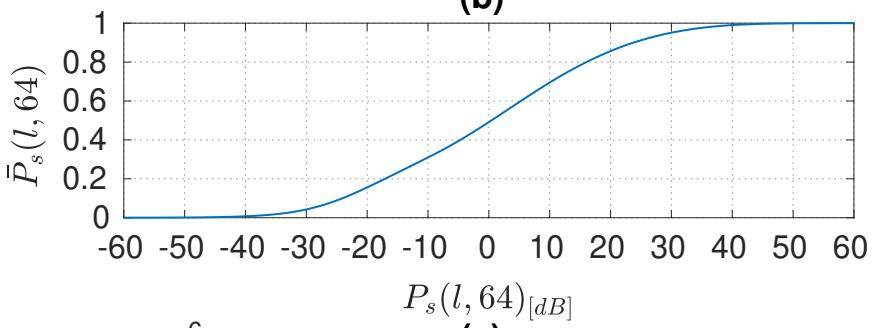

(c)

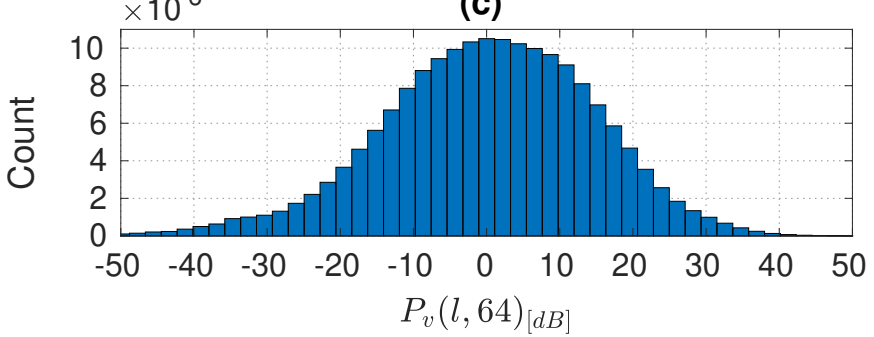

(d)

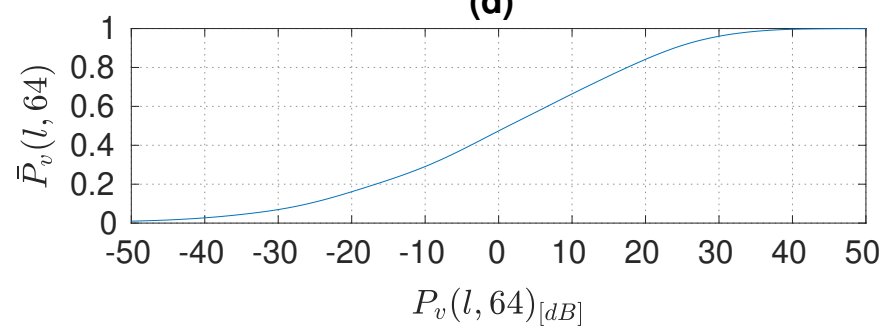

Figure 2: (Color online) The distribution of (a) $P_{s}(l, 64)_{[\mathrm{dB}]}$ and (c) $P_{v}(l, 64)_{[\mathrm{dB}]}$. The CDF of (b) $P_{s}(l, 64)_{[\mathrm{dB}]}$ and $(\mathrm{d}) P_{v}(l, 64)_{[\mathrm{dB}]}$, where the sample mean and variance were found over the sample of the training set ${ }^{1}$.

and $\left(\mu_{v}, \sigma_{v}^{2}\right)$ for each frequency bin, $m$ were found over a sample of the training set ${ }^{1}$. It is also shown in Figs. 2(b) and $(\mathrm{d})$ that the CDFs of $P_{s}(l, 64)_{[\mathrm{dB}]}$ and $P_{v}(l, 64)_{[\mathrm{dB}]}$ computed over the training sample ${ }^{1}$ were used to compress the dynamic range of $P_{s}(l, 64)_{[\mathrm{dB}]}$ and $P_{v}(l, 64)_{[\mathrm{dB}]}$, respectively. In light of this observation, the CDFs of $P_{s}(l, m)_{[\mathrm{dB}]}$ and $P_{v}(l, m)_{[\mathrm{dB}]}$ are used to compress the dynamic range of the training targets, as demonstrated in

\footnotetext{
${ }^{1} 2500$ randomly selected clean speech recordings were mixed with 2500 randomly selected noise recordings from the training set (Section 5.1) with SNR levels: $-10 \mathrm{~dB}$ to $+20 \mathrm{~dB}$ in $1 \mathrm{~dB}$ increments, giving 2500 noisy speech signals. For each frequency bin, $m$, the sample mean and variances, $\left(\mu_{s}, \sigma_{s}^{2}\right)$ and $\left(\mu_{v}, \sigma_{v}^{2}\right)$ were computed from 2500 concatenated clean speech recordings and scaled noises, respectively.
}

DeepLPC-ResNet-TCN (Roy et al., 2021a):

$$
\begin{aligned}
& \bar{P}_{s}(l, m)=\frac{1}{2}\left[1+\operatorname{erf}\left(\frac{P_{s}(l, m)_{[\mathrm{dB}]}-\mu_{s}}{\sigma_{s} \sqrt{2}}\right)\right], \\
& \bar{P}_{v}(l, m)=\frac{1}{2}\left[1+\operatorname{erf}\left(\frac{P_{v}(l, m)_{[\mathrm{dB}]}-\mu_{v}}{\sigma_{v} \sqrt{2}}\right)\right] .
\end{aligned}
$$

To facilitate the training, the final training target, $\boldsymbol{\zeta}_{l}$ of size $M \times 2$ is formed by concatenating $\bar{P}_{s}(l, m)$ and $\bar{P}_{v}(l, m)$ as:

$$
\begin{aligned}
\boldsymbol{\zeta}_{l}=\{ & \bar{P}_{s}(l, 0), \bar{P}_{s}(l, 1), \ldots, \bar{P}_{s}(l, M-1), \bar{P}_{v}(l, 0), \\
& \left.\bar{P}_{v}(l, 1), \ldots, \bar{P}_{v}(l, M-1)\right\}
\end{aligned}
$$

Then $\boldsymbol{\zeta}_{l}$ is used as the final training target in the supervised LPC estimation framework (Fig. 1). During inference, $\hat{\zeta}_{l}$ is first split into $\hat{\bar{P}}_{s}(l, m)$ and $\hat{\bar{P}}_{v}(l, m)$ and compute the LPC-PS as:

$$
\begin{aligned}
& \hat{P}_{s}(l, m)=10^{\left(\left(\sigma_{s} \sqrt{2} \operatorname{erf}^{-1}\left(2 \hat{\bar{P}}_{s}(l, m)-1\right)+\mu_{s}\right) / 10\right)} \\
& \hat{P}_{v}(l, m)=10^{\left(\left(\sigma_{v} \sqrt{2} \operatorname{erf}^{-1}\left(2 \hat{\bar{P}}_{v}(l, m)-1\right)+\mu_{v}\right) / 10\right)} .
\end{aligned}
$$

The $\mid$ IDFT $\mid$ of $\hat{P}_{s}(l, m)$ and $\hat{P}_{v}(l, m)$ gives an estimate of the autocorrelation matrices, $\widehat{R}_{s s}(\tau)$ and $\widehat{R}_{v v}(\tau)$. As in DeepLPC-ResNet-TCN (Roy et al., 2021a, eq. (26)(27)), we construct Yule-Walker equations with the estimated $\widehat{R}_{s s}(\tau)$ and $\widehat{R}_{v v}(\tau)$ followed by solving them using the Levinson-Durbin recursion (Vaseghi, 2006, Chapter 8), yielding $\left(\left\{\hat{a}_{i}\right\}, \hat{\sigma}_{w}^{2}\right)(p=16)$ and $\left(\left\{\hat{b}_{k}\right\}, \hat{\sigma}_{u}^{2}\right)(q=16)$.

\subsection{PS training target}

As like LPC-PS in Section 3.2, the PS of clean speech and noise (denoted as $\lambda_{s}(l, m)$ and $\lambda_{v}(l, m)$ ) can also be used as the training targets for supervised LPC estimation. For this purpose, in the training stage, $\lambda_{s}(l, m)$ and $\lambda_{v}(l, m)$ are computed directly from the squared magnitude of the clean speech and noise spectral components (single-sided) as: $\lambda_{s}(l, m)=|S(l, m)|^{2}$ and $\lambda_{v}(l, m)=$ $|V(l, m)|^{2}$. Motivated by the dynamic range compression of LPC-PS in Section 3.2, we also utilise the CDF of the training targets (in $\mathrm{dB}), \lambda_{s}(l, m)_{[\mathrm{dB}]}$ and $\lambda_{v}(l, m)_{[\mathrm{dB}]}$ to compress their dynamic range to the interval $[0,1]$, where $\lambda_{s}(l, m)_{[\mathrm{dB}]}=10 \log _{10}\left(\lambda_{s}(l, m)\right)$ and $\lambda_{v}(l, m)_{[\mathrm{dB}]}=$ $10 \log _{10}\left(\lambda_{v}(l, m)\right)$. For instance, we observed in Figs. 3(a) and $(\mathrm{c})$ that $\lambda_{s}(l, 64)_{[\mathrm{dB}]}$ and $\lambda_{v}(l, 64)_{[\mathrm{dB}]}$ follow a Gaussian distribution. In light of the analysis, we assume that $\lambda_{s}(l, m)_{[\mathrm{dB}]}$ and $\lambda_{v}(l, m)_{[\mathrm{dB}]}$ are also distributed normally with mean, $\mu_{s}$ and $\mu_{v}$, and variance $\sigma_{s}^{2}$ and $\sigma_{v}^{2}$, respectively $\left(\lambda_{s}(l, m)_{[\mathrm{dB}]} \sim \mathcal{N}\left(\mu_{s}, \sigma_{s}^{2}\right)\right.$ and $\left.\lambda_{v}(l, m)_{[\mathrm{dB}]} \sim \mathcal{N}\left(\mu_{v}, \sigma_{v}^{2}\right)\right)$. The statistics of $\lambda_{s}(l, m)_{[\mathrm{dB}]}$ and $\lambda_{v}(l, m)_{[\mathrm{dB}]}$, i.e., $\left(\mu_{s}, \sigma_{s}^{2}\right)$ and $\left(\mu_{v}, \sigma_{v}^{2}\right)$ for each frequency bin, $m$ were found over a sample of the training set ${ }^{1}$. The CDFs of $\lambda_{s}(l, 64)_{[\mathrm{dB}]}$ and $\lambda_{v}(l, 64)_{[\mathrm{dB}]}$ computed over the training set ${ }^{1}$ (Fig. 3 (b) and (d)) are used to compress the dynamic range of $\lambda_{s}(l, 64)_{[\mathrm{dB}]}$ and $\lambda_{v}(l, 64)_{[\mathrm{dB}]}$, respectively. Thus, the CDFs 


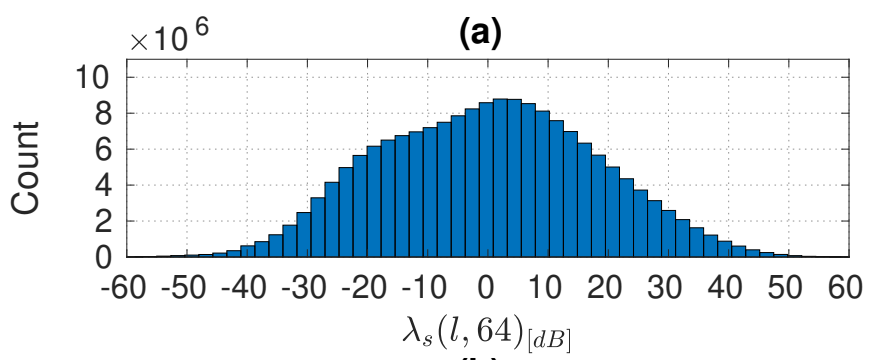

(b)

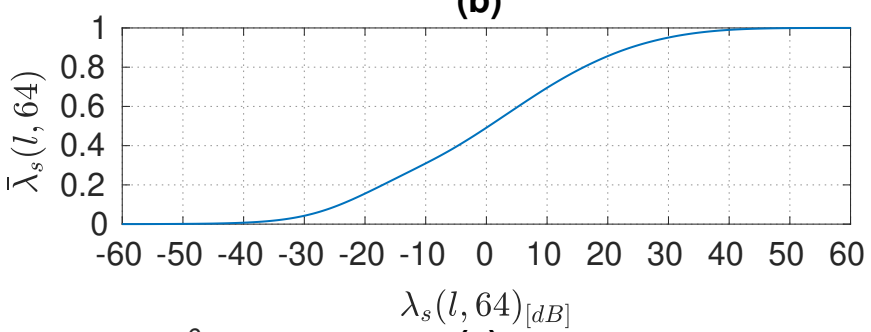

(c)

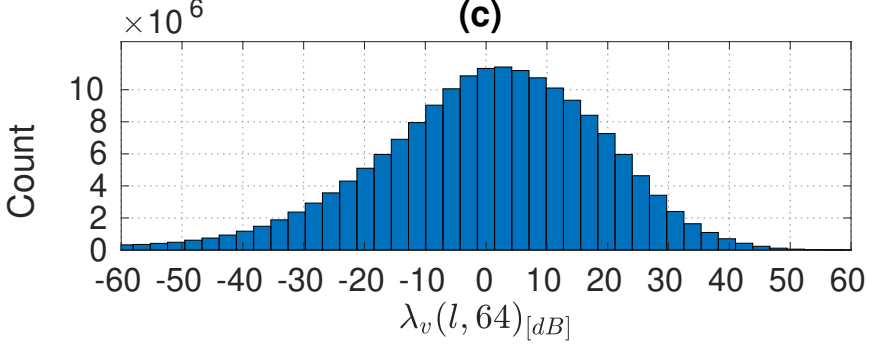

(d)

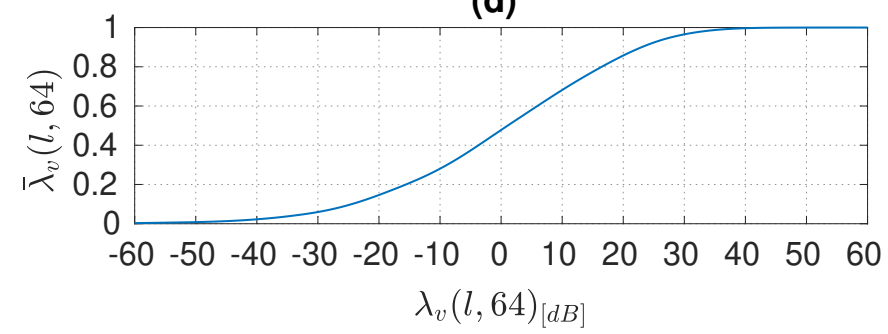

Figure 3: (Color online) The distribution of (a) $\lambda_{s}(l, 64)_{[\mathrm{dB}]}$ and (c) $\lambda_{v}(l, 64)_{[\mathrm{dB}]}$. The CDF of (b) $\lambda_{s}(l, 64)_{[\mathrm{dB}]}$ and $(\mathrm{d}) \lambda_{v}(l, 64)_{[\mathrm{dB}]}$, where the sample mean and variance were found over the sample of the training set ${ }^{1}$.

of $\lambda_{s}(l, m)_{[\mathrm{dB}]}$ and $\lambda_{v}(l, m)_{[\mathrm{dB}]}$ are used to compress the dynamic range of training targets, as in equations ((27)$(28))$ :

$$
\begin{aligned}
& \bar{\lambda}_{s}(l, m)=\frac{1}{2}\left[1+\operatorname{erf}\left(\frac{\lambda_{s}(l, m)_{[\mathrm{dB}]}-\mu_{s}}{\sigma_{s} \sqrt{2}}\right)\right], \\
& \bar{\lambda}_{v}(l, m)=\frac{1}{2}\left[1+\operatorname{erf}\left(\frac{\lambda_{v}(l, m)_{[\mathrm{dB}]}-\mu_{v}}{\sigma_{v} \sqrt{2}}\right)\right] .
\end{aligned}
$$

To facilitate the training, for $l^{\text {th }}$ frame, the final training target, $\zeta_{l}$ of size $M \times 2$ is formed by concatenating $\bar{\lambda}_{s}(l, m)$ and $\bar{\lambda}_{v}(l, m)$ as:

$$
\begin{aligned}
\boldsymbol{\zeta}_{l}= & \left\{\bar{\lambda}_{s}(l, 0), \bar{\lambda}_{s}(l, 1), \ldots, \bar{\lambda}_{s}(l, M-1), \bar{\lambda}_{v}(l, 0),\right. \\
& \left.\bar{\lambda}_{v}(l, 1), \ldots, \bar{\lambda}_{v}(l, M-1)\right\} .
\end{aligned}
$$

Then $\boldsymbol{\zeta}_{l}$ is used as the training target for the LPC estimation framework (Fig. 1). During inference, $\hat{\boldsymbol{\zeta}}_{l}$ is first split into $\hat{\bar{\lambda}}_{s}(l, m)$ and $\hat{\bar{\lambda}}_{v}(l, m)$ and compute the PSs as:

$$
\begin{aligned}
& \hat{\lambda}_{s}(l, m)=10^{\left(\left(\sigma_{s} \sqrt{2} \operatorname{erf}^{-1}\left(2 \hat{\bar{\lambda}}_{s}(l, m)-1\right)+\mu_{s}\right) / 10\right)}, \\
& \hat{\lambda}_{v}(l, m)=10^{\left(\left(\sigma_{v} \sqrt{2} \operatorname{erf}^{-1}\left(2 \hat{\bar{\lambda}}_{v}(l, m)-1\right)+\mu_{v}\right) / 10\right)} .
\end{aligned}
$$

The $|\mathrm{IDFT}|$ of $\hat{\lambda}_{s}(l, m)$ and $\hat{\lambda}_{v}(l, m)$ yields an estimate of the autocorrelation matrices, $\widehat{R}_{s s}(\tau)$ and $\widehat{R}_{v v}(\tau)$. As demonstrated in (Roy et al., 2021a, eq. (26)-(27)), we construct Yule-Walker equations with the estimated $\widehat{R}_{s s}(\tau)$ and $\widehat{R}_{v v}(\tau)$ followed by solving them using the LevinsonDurbin recursion (Vaseghi, 2006, Chapter 8), yielding $\left(\left\{\hat{a}_{i}\right\}\right.$, $\left.\hat{\sigma}_{w}^{2}\right)(p=16)$ and $\left(\left\{\hat{b}_{k}\right\}, \hat{\sigma}_{u}^{2}\right)(q=16)$.

\subsection{MS training target}

Motivated by CCED-AKF (Roy and Paliwal, 2020a), in this study, the MS of clean speech and noise signal (denoted as $C_{s}(l, m)$ and $\left.C_{v}(l, m)\right)$ can also be used as the training targets for supervised LPC estimation. In the training stage, $C_{s}(l, m)$ and $C_{v}(l, m)$ are computed directly from the magnitude of the clean speech and noise spectral components as: $C_{s}(l, m)=|S(l, m)|$ and $C_{v}(l, m)=$ $|V(l, m)|$. As demonstrated in CCED-AKF (Roy and Paliwal, 2020a), we have also used the min-max normalization (Han et al., 2011, section 3.5.2) to compress the dynamic range of $C_{s}(l, m)$ and $C_{v}(l, m)$ as:

$$
\begin{aligned}
& \bar{C}_{s}(l, m)=\frac{C_{s}(l, m)-S_{\min }(m)}{S_{\max }(m)-S_{\min }(m)}, \\
& \bar{C}_{v}(l, m)=\frac{C_{v}(l, m)-V_{\min }(m)}{V_{\max }(m)-V_{\min }(m)},
\end{aligned}
$$

where $\left(S_{\max }, S_{\min }\right)$ and $\left(V_{\max }, V_{\min }\right)$ for each frequency bin, $m$ were found over a sample of the training set ${ }^{1}$.

As in Section 3.3, for $l^{\text {th }}$ frame, the final training target, $\boldsymbol{\zeta}_{l}$ of size $M \times 2$ is formed by concatenating $\bar{C}_{s}(l, m)$ and $\bar{C}_{v}(l, m)$ as:

$$
\begin{aligned}
\zeta_{l}=\left\{\bar{C}_{s}(l, 0), \bar{C}_{s}(l, 1), \ldots, \bar{C}_{s}(l, M-1), \bar{C}_{v}(l, 0),\right. \\
\\
\left.\bar{C}_{v}(l, 1), \ldots, \bar{C}_{v}(l, M-1)\right\} .
\end{aligned}
$$

Then $\boldsymbol{\zeta}_{l}$ is used as the training target for the LPC estimation framework (Fig. 1). During inference, $\hat{\boldsymbol{\zeta}}_{l}$ is first split into $\hat{\bar{C}}_{s}(l, m)$ and $\hat{\bar{C}}_{v}(l, m)$. Then compute the clean speech and noise MSs from $\hat{\bar{C}}_{s}(l, m)$ and $\hat{\bar{C}}_{v}(l, m)$ using the inverse min-max normalization as (Han et al., 2011, section 3.5.2):

$$
\begin{aligned}
& \hat{C}_{s}(l, m)=S_{\min }(m)+\hat{\bar{C}}_{s}(l, m)\left(S_{\max }(m)-S_{\min }(m)\right), \\
& \hat{C}_{v}(l, m)=V_{\min }(m)+\hat{\bar{C}}_{v}(l, m)\left(V_{\max }(m)-V_{\min }(m)\right) .
\end{aligned}
$$

By taking the square of $\hat{C}_{s}(l, m)$ and $\hat{C}_{v}(l, m)$ followed by $|\mathrm{IDFT}|$ of them yields the autocorrelation matrices, 
$\widehat{R}_{s s}(\tau)$ and $\widehat{R}_{v v}(\tau)$. Then compute $\left(\left\{\hat{a}_{i}\right\}, \hat{\sigma}_{w}^{2}\right)(p=16)$ and $\left(\left\{\hat{b}_{k}\right\}, \hat{\sigma}_{u}^{2}\right)(q=16)$ from $\widehat{R}_{s s}(\tau)$ and $\widehat{R}_{v v}(\tau)$ using the similar procedure as in Section 3.3.

\section{Deep neural network}

The DNN methods-ResNet-TCN and MHANet used in this study are briefly described below.

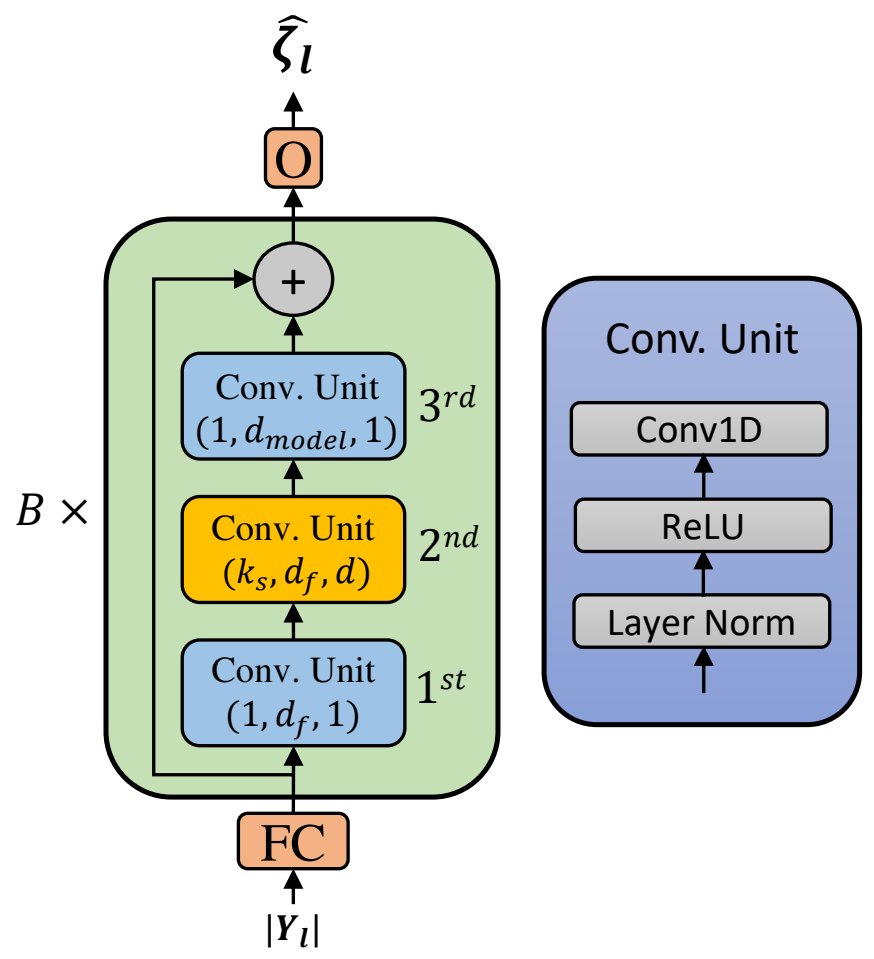

Figure 4: (Color online) ResNet-TCN. The kernel size, output size, and dilation rate for each convolutional unit is denoted as (kernel size, output size, dilation rate).

\subsection{ResNet-TCN}

The ResNet-TCN from DeepLPC framework (Roy et al., $2021 \mathrm{a}$ ) is also used in this study to estimate $\boldsymbol{\zeta}_{l}$ (Equations (24), (29), (34), and (39)) from $\left|\boldsymbol{Y}_{l}\right|$. The ResNetTCN is shown in Fig. 4. For each training target (Section 3), the input, $\left|\boldsymbol{Y}_{l}\right|$ is first passed through FC, a fullyconnected layer of size $d_{\text {model }}$, followed by layer normalization (LN) (Ba et al., 2016) and the rectified linear unit (ReLU) activation function (He et al., 2015). FC is followed by $B$ bottleneck residual blocks, where $j \epsilon\{1,2, \ldots, B\}$ is the block index. Each block comprise of three onedimensional causal convolutional units. Each convolutional unit (CU) is pre-activated by LN (Ba et al., 2016) followed by the ReLU activation function (He et al., 2015). The kernel size, output size, and dilation rate for each convolutional unit is denoted as (kernel size, output size, dilation rate). The first and third CUs in each block has a kernel size of one, whilst the second convolutional unit has a kernel size of $k_{s}$. The output size of the first and second $\mathrm{CU}$ is $d_{f}$, while the third one is $d_{\text {model }}$. A dilation rate one is set for the first and the third CU, which is $d$ for the second CU. The second CU provides a contextual field over previous time steps. The dilation rate, $d$ is cycled as the block index $j$ increases as: $d=2^{\left(j-1 \bmod \left(\log _{2}(D)+1\right)\right.}$, where mod is the modulo operation, and $D$ is the maximum dilation rate. In (Roy et al., 2021a, Fig. 5), it was demonstrated that the contextual field gained by the use of causal dilated CUs. The last residual block is followed by the output layer, $\mathbf{O}$, which is a fully-connected layer with sigmoidal units. The $\mathbf{O}$ layer gives an estimate of $\hat{\boldsymbol{\zeta}}_{l}$.

For LPC-PS, PS, and MS training targets, the hyperparameters used in DeepLPC (Roy et al., 2021a) also used in this study: $d_{\text {model }}=256, d_{f}=64, B=40$, $k_{s}=3$, and $D=16$. With this set of hyperparameters, the ResNet-TCN exhibits approximately 2.1 million parameters. For LSF, all the above hyperparameters were used except $d_{f}=34$ gives around 1.91 million parameters. Section 5.2 details the training strategy of the ResNet-TCN.

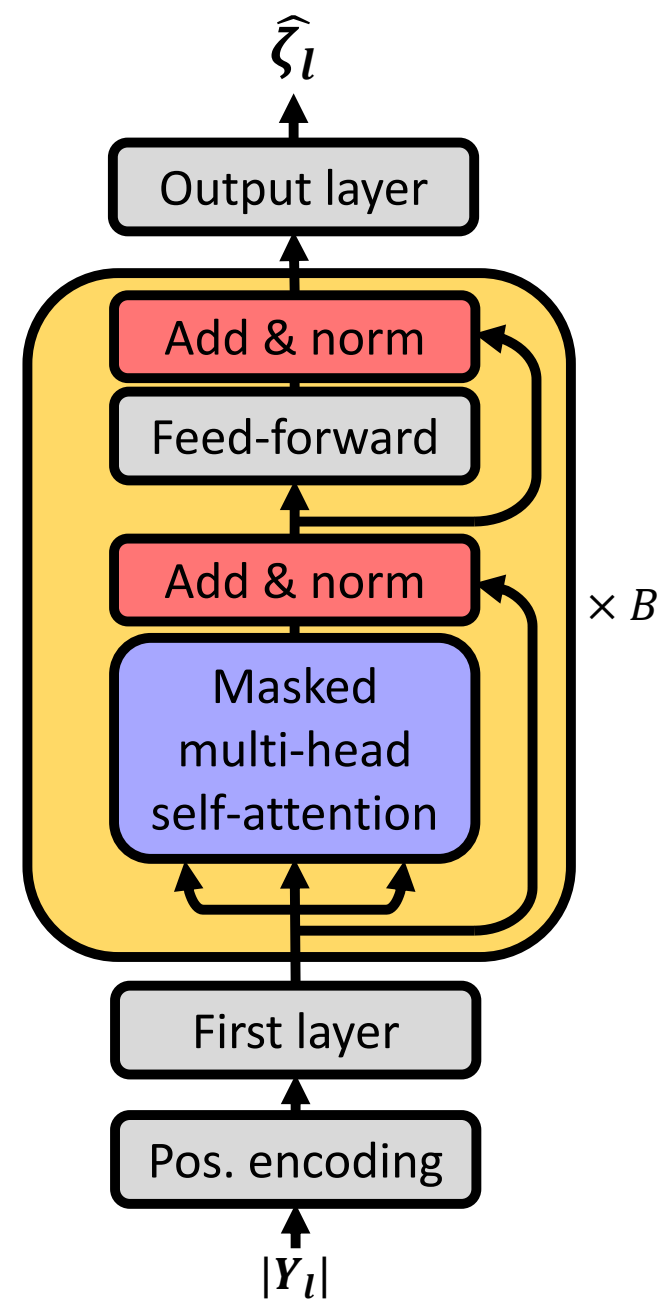

Figure 5: (Color online) MHANet within the multi-head attention network for LPC estimation. 


\section{2. $M H A N e t$}

The MHANet from (Nicolson and Paliwal, 2020) is used in this study. Since MHANet is a large framework, we briefly summarize it in this section. For a detailed description of MHANet, we refer the reader to (Nicolson and Paliwal, 2020). The simplest form of MHANet is shown in Fig. 5. The processing steps of the MHANet from input to output are described as follows. For each training target, $\boldsymbol{\zeta}_{l}$ (Equations (24), (29), (34), and (39)), $\left|\boldsymbol{Y}_{l}\right|$ is fed as the input to MHANet. The first layer in MHANet is used to project the input to a size of $d_{\text {model }}$. As in (Nicolson and Paliwal, 2019), the first layer is formed as: $\max \left(0, \mathrm{LN}\left(|\boldsymbol{X}| \boldsymbol{W}^{I}+\boldsymbol{b}_{s}^{I}\right)\right)$, where $\mathrm{LN}$ is frame-wise layer normalisation (Ba et al., 2016), $\boldsymbol{W}^{I} \in \mathbb{R}^{M \times d_{\text {model }} \text {, and }}$ $\boldsymbol{b}_{s}^{I} \in \mathbb{R}^{d_{\text {model }}}$. Next, the positional encoding from (Nicolson and Paliwal, 2020) is added after the first layer, where the time-frame index indicates the position. The position encoding is learned using weight matrix $\mathbf{W}_{p}$, with a maximum length of 2048 time-frames (i.e. $\mathbf{W}_{p} \in \mathbb{R}^{2048 \times 256}$ ). This is followed by $B$ cascading blocks. Each block includes an MHA module, a two-layer feed-forward neural network (FNN) (Xu et al., 2014), residual connections (He et al., 2016), and frame-wise LN (Ba et al., 2016). For the detailed description of the blocks, we refer the reader to (Nicolson and Paliwal, 2020, Section 3.1). The last block is followed by the output layer, which is a sigmoidal feedforward layer as in (Nicolson and Paliwal, 2019). The output layer gives an estimate of $\hat{\zeta}$.

For all training targets, the hyperparameters used in DeepLPC-MHANet (Roy et al., 2021b) also used in this study: $B=5, d_{f}=1024, d_{\text {model }}=256, H=8, P_{d r o p}=$ 0.0 , and $\Gamma=40000$. With this set of hyperparameters, the MHANet exhibits approximately 4.27 million parameters. Section 5.2 details the training strategy of the MHANet.

\section{Experimental setup}

\subsection{Training and validation set}

The noisy speech for the training and validation sets are formed from clean speech and noise recordings. For the clean speech recordings, the train-clean-100 set of the Librispeech corpus (Panayotov et al., 2015) (28539), the CSTR VCTK corpus (Veaux et al., 2017) (42015), and the $s i^{*}$ and $s x^{*}$ training sets of the TIMIT corpus (Garofolo et al., 1993) (3696) were used, giving a total 74250 clean speech recordings. To form the validation set, $5 \%$ of the clean speech recordings (3713) are randomly selected. Thus, 70537 of the clean speech recordings are used for the training set. For the noise recordings, the QUT-NOISE dataset (Dean et al., 2010), the Nonspeech dataset $(\mathrm{Hu}, 2004)$, the Environmental Background Noise dataset (Saki and Kehtarnavaz, 2016; Saki et al., 2016), the noise set from the MUSAN corpus (Snyder et al., 2015), multiple FreeSound packs (https://freesound.org/) ${ }^{2}$, and

\footnotetext{
${ }^{2}$ Freesound packs that were used: 147, 199, 247, 379, 622, 643 1 133, 1563, 1840, 2432, 4366, 4 439, 15 046, 15 598, 21558 .
}

coloured noise recordings (with a value ranging from 2 to 2 in increments of 0.25 ) were used, giving a total of 16243 noise recordings. For the validation set, $5 \%$ of the noise recordings (813) are randomly selected. The remaining 15430 noise recordings are used for the training set. All the clean speech and noise recordings are single-channel with a sampling frequency of $16 \mathrm{kHz}$. To create the noisy speech for the validation set, each of the 3713 clean speech recordings is corrupted by a random section of a randomly selected noise recording (from the set of 813 noise recordings) at a randomly selected SNR level $(-10$ to $+20 \mathrm{~dB}$, in $1 \mathrm{~dB}$ increments). The noisy speech for the training set was created using the method described in Section 5.2.

\subsection{Training strategy}

The following training strategy was employed for training ResNet-TCN and MHANet:

- Mean squared error is used as the loss function.

- The Adam optimiser (Kingma and Ba, 2014) with $\beta_{1}=0.9, \beta_{2}=0.98$, and $\epsilon=10^{-9}$ is used for stochastic gradient descent optimisation, where the learning rate, $\alpha_{r}$, is controlled over the course of training as in (Vaswani et al., 2017):

$$
\alpha_{r}=d_{\text {model }}^{-0.5} \cdot \min \left(\gamma^{-0.5}, \gamma \cdot \Gamma^{-1.5}\right),
$$

where $\gamma$ is the training step and $\Gamma$ is the number of warmup steps.

- Gradients are clipped between $[-1,1]$.

- The number of training examples in an epoch is equal to the number of clean speech recordings used in the training set, i.e., 70537.

- A mini-batch size of eight training examples is used.

- The noisy speech signals are generated on the fly as follows: each clean speech recording is randomly selected and corrupted with a randomly selected noise recording at a randomly selected SNR level (-10 to $+20 \mathrm{~dB}$, in $1 \mathrm{~dB}$ increments).

- Epoch 150 is used to train both the ResNet-TCN and MHANet.

\subsection{Test set}

For the objective experiments, 30 clean speech utterances belonging to six speakers (three male and three female) are taken from the NOIZEUS corpus (Loizou, 2013, Chapter 12). The noisy speech for the test set is formed by mixing the clean speech with real-world non-stationary (voice babble, street) and colored (factory and f16) noise recordings selected from (Saki and Kehtarnavaz, 2016; Saki et al., 2016) at multiple SNR levels varying from $-5 \mathrm{~dB}$ to $+15 \mathrm{~dB}$, in $5 \mathrm{~dB}$ increments. This provides 30 examples per condition with 20 total conditions. All the clean speech and noise recordings are single-channel with a sampling frequency of $16 \mathrm{kHz}$. Note that the speech and the noise recordings in the test set are different from those used in the training and validation sets. 


\subsection{SD level evaluation}

The frame-wise spectral distortion (SD) (dB) (Gray and Markel, 1976) is used to evaluate the accuracy of LPC estimates obtained using ResNet-TCN and MHANet for the training targets; LSF, LPC-PS, PS, and MS. Specifically, the estimated clean speech LPCs are evaluated. SD for $l^{\text {th }}$ frame, denoted by $D_{l}($ in $\mathrm{dB})$ is defined as the rootmean-square-difference between the LPC-PS estimate in $\mathrm{dB}, \hat{P}_{s}(l, m)_{[d B]}$, and the oracle case in $\mathrm{dB}, P_{s}(l, m)_{[d B]}$ as (Gray and Markel, 1976):

$$
D_{l}=\sqrt{\frac{1}{M} \sum_{m=0}^{M-1}\left[P_{s}(l, m)_{[d B]}-\hat{P}_{s}(l, m)_{[d B]}\right]^{2}}
$$

\subsection{Speech enhancement methods}

We also evaluate the performance of the LPC estimation in speech enhancement context. For this purpose, the specifications of competitive SEAs are as follows:

1. Noisy: speech corrupted with additive noise.

2. Oracle-AKF: AKF, where $\left(\left\{a_{i}\right\}, \sigma_{w}^{2}\right)$ and $\left(\left\{b_{k}\right\}, \sigma_{u}^{2}\right)$ are computed from the clean speech and the noise signal, where $p=16, q=16$, window length=32 ms, frame shift $=16 \mathrm{~ms}$, and a rectangular window is used for framing.

3. AKF constructed with speech and noise LPC parameters derived from the training targets - LSF, LPCPS, PS, and MS using ResNet-TCN and MHANet. Thus, there are eight AKF methods, where $p=16$, $q=16$, window length $=32 \mathrm{~ms}$, frame shift $=16 \mathrm{~ms}$, and a rectangular window is used for framing.

4. Deep Xi-ResNet-TCN-MMSE-LSA: Deep Xi-ResNetTCN (Zhang et al., 2020) estimates the a priori SNR for the MMSE-LSA estimator (Ephraim and Malah, $1985)$, where $w_{f}=32 \mathrm{~ms}, s_{f}=16 \mathrm{~ms}$, and a squareroot-Hann window is used for analysis and synthesis.

Table 1: Objective measures, what each assesses, and the range of their scores. For each measure, higher is better.

\begin{tabular}{ccc}
\hline Measure & Assesses & Range \\
\hline CSIG (Hu and Loizou, 2008) & Quality & {$[1,5]$} \\
CBAK (Hu and Loizou, 2008) & Quality & {$[1,5]$} \\
COVL (Hu and Loizou, 2008) & Quality & {$[1,5]$} \\
PESQ (Rix et al., 2001) & Quality & {$[-0.5,4.5]$} \\
STOI (Taal et al., 2011) & Intelligibility & {$[0,100] \%$} \\
SI-SDR (Roux et al., 2019) & Quality & {$[-\infty, \infty]$} \\
SegSNR (Mermelstein, 1979) & Quality & {$[-\infty, \infty]$} \\
\hline
\end{tabular}

\subsection{Objective quality and intelligibility measures}

Objective measures are used to evaluate the quality and intelligibility of the enhanced speech with respect to the corresponding clean speech. Table 1 shows the objective quality and intelligibility measures used in this study.
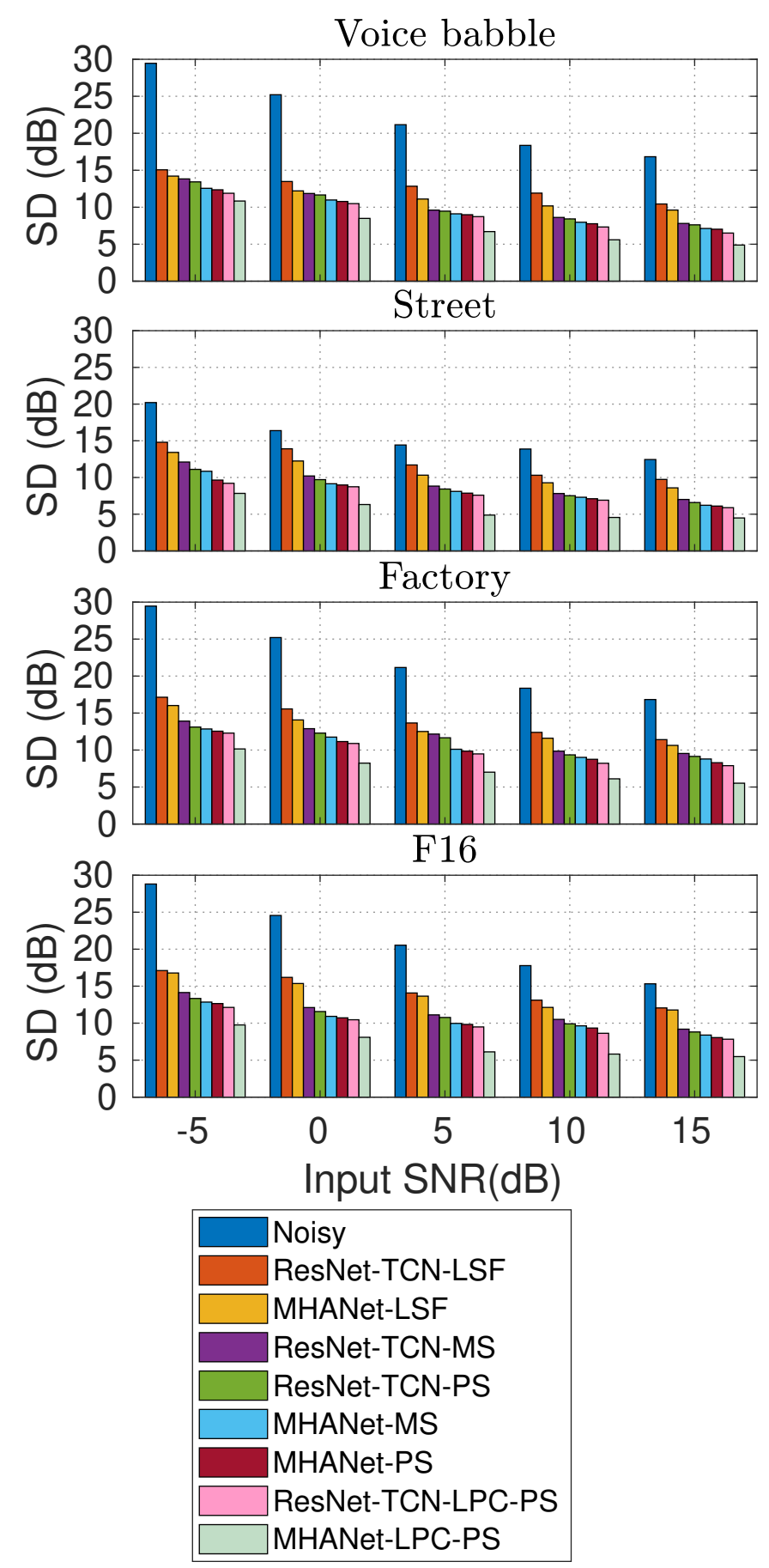

Figure 6: (Color online) Average SD (dB) level for each SEA found over all frames for each test condition in Section 5.3.

\subsection{Subjective evaluation for speech enhancement}

The subjective evaluation was carried out through a series of blind AB listening tests (Paliwal et al., 2010, Section 3.3.4). The test is performed on utterance sp05 ("Wipe the grease off his dirty face") from NOIZEUS corpus (Loizou, 2013, Chapter 12) corrupted by $5 \mathrm{~dB}$ voice babble noise in Section 5.3. In this test, the enhanced speech produced by ten SEAs as well as the corresponding clean speech and 
Table 2: Mean objective scores on NOIZEUS corpus in terms of CSIG, CBAK, COVL, PESQ, STOI, SegSNR, and SI-SDR. Apart from Oracle-AKF, the highest score amongst the competing methods for each measure is given in boldface.

\begin{tabular}{cccccccc}
\hline Methods & CSIG & CBAK & COVL & PESQ & STOI & SegSNR & SI-SDR \\
\hline Noisy speech & 2.41 & 2.27 & 2.12 & 1.64 & 67.67 & 0.89 & 6.39 \\
ResNet-TCN-LSF-AKF & 2.91 & 2.72 & 2.51 & 2.03 & 74.73 & 7.14 & 10.19 \\
MHANet-LSF-AKF & 2.97 & 2.76 & 2.55 & 2.11 & 76.96 & 7.35 & 11.52 \\
ResNet-TCN-MS-AKF & 3.23 & 2.81 & 2.70 & 2.25 & 79.17 & 7.45 & 12.27 \\
ResNet-TCN-PS-AKF & 3.32 & 2.89 & 2.77 & 2.29 & 80.29 & 7.53 & 12.69 \\
Deep Xi-ResNet-TCN-MMSE-LSA & 3.38 & 3.02 & 2.81 & 2.34 & 81.53 & 7.67 & 13.39 \\
MHANet-MS-AKF & 3.39 & 3.06 & 2.84 & 2.38 & 82.59 & 7.73 & 13.52 \\
MHANet-PS-AKF & 3.42 & 3.09 & 2.88 & 2.42 & 83.84 & 7.83 & 13.67 \\
ResNet-TCN-LPC-PS-AKF & 3.49 & 3.17 & 2.95 & 2.51 & 85.34 & 8.78 & 14.44 \\
MHANet-LPC-PS-AKF & $\mathbf{3 . 6 6}$ & $\mathbf{3 . 3 2}$ & $\mathbf{3 . 1 4}$ & $\mathbf{2 . 6 3}$ & $\mathbf{8 8 . 6 4}$ & $\mathbf{9 . 8 1}$ & $\mathbf{1 5 . 4 8}$ \\
Oracle-AKF & 4.21 & 4.07 & 3.97 & 2.74 & 95.61 & 10.87 & 16.43 \\
\hline
\end{tabular}

noisy speech signals were played as stimuli pairs to the listeners. Specifically, the test is performed on a total of 132 stimuli pairs played in a random order to each listener, excluding the comparisons between the same method. The listener prefers the first or second stimuli, which is perceptually better, or a third response indicating no difference is found between them. For a pairwise scoring, 100\% award is given to the preferred method, $0 \%$ to the other, and $50 \%$ for the similar preference response. The participants could re-listen to stimuli if required. Ten English speaking listeners participate in the blind $\mathrm{AB}$ listening tests ${ }^{3}$. The average of the preference scores given by the listeners termed as mean subjective preference score (\%), which is used to compare the efficiency among the SEAs.

\section{Results and discussion}

\subsection{SD level comparison}

The average SD levels (in $\mathrm{dB}$ ) attained by the ResNetTCN and MHANet for training targets - LSF, LPC-PS, $\mathrm{PS}$, and MS are shown in Fig. 6. It can be seen that the MHANet-LPC-PS is able to produce the lowest SD levels than other competing methods for both real-world nonstationary as well as colored noise conditions. Amongst other competing methods, ResNet-TCN-LPC-PS produced the next lowest SD level. The SD levels for noisy speech indicate the upper bounds of the SD level. In light of the comparative study, it is evident to say that the best training target and DNN method for accurate LPC estimation in practice are LPC-PS and MHANet, respectively. The lowest SD level attained by the MHANet-LPC-PS will be of benefit to the AKF for speech enhancement.

\subsection{Objective evaluation}

In this section, we analyze the performance improvement among the competing methods (Section 5.5) for the

\footnotetext{
${ }^{3}$ The AB listening tests were conducted with approval from the Griffith University's Human Research Ethics Committee: database protocol number 2018/671.
}

objective measures described in Table 1. The mean objective evaluation results for the NOIZEUS corpus are shown in Tables 2, respectively. It can be seen that Oracle-AKF produces the highest scores for all measures, which can be thought of as the upper boundary of performance. Noisy speech produced the lowest scores for all measures, indicating the lower boundary of performance. When comparing the performance of the MHANet-LPC-PS-AKF, it shows a consistent CSIG, CBAK, COVL, PESQ, STOI, SegSNR, and SI-SDR score improvement over the competing methods. This demonstrates that MHANet-LPCPS-AKF produces enhanced speech at a higher quality and intelligibility than any competing methods.

Figures 7 and 8 show the PESQ and STOI scores, respectively, of each SEA for multiple conditions. The MHANet-LPC-PS-AKF method produced higher PESQ and STOI scores than other competing SEAs for each condition. This demonstrates that the MHANet-LPC-PSAKF method is able to produce higher objective quality and intelligibility scores than the competing methodsacross multiple SNR levels and noise sources.

\subsection{Subjective evaluation by AB listening test}

The mean subjective preference score $(\%)$ for each SEA is shown in Fig. 9. It can be seen that the MHANet-LPCPS-AKF method is widely preferred (around 73\%) by the listeners to that of the competing methods, apart from the clean speech (100\%) and the Oracle-AKF method (83\%). Also, ResNet-TCN-LPC-PS-AKF is found the next best preferred (71\%), with MHANet-PS-AKF (69\%), MHANetMS-AKF (67\%), Deep Xi-ResNet-TCN-MMSE-LSA (64\%), ResNet-TCN-PS-AKF (58\%), and ResNet-TCN-MS-AKF (55\%). Also, MHANet-LSF-AKF (49\%) and ResNet-TCNLSF-AKF (44\%) achieve the lowest preference scores among the competing methods. It is due to the MHANet-LSF and ResNet-TCN-LSF methods produced higher SD levels in the estimated speech LPCs (Fig. 6). In light of the blind $\mathrm{AB}$ listening tests, it is evident to say that the enhanced speech produced by the MHANet-LPC-PS-AKF exhibits the best perceived quality amongst all tested methods for the tested condition specified in Section 5.7. 

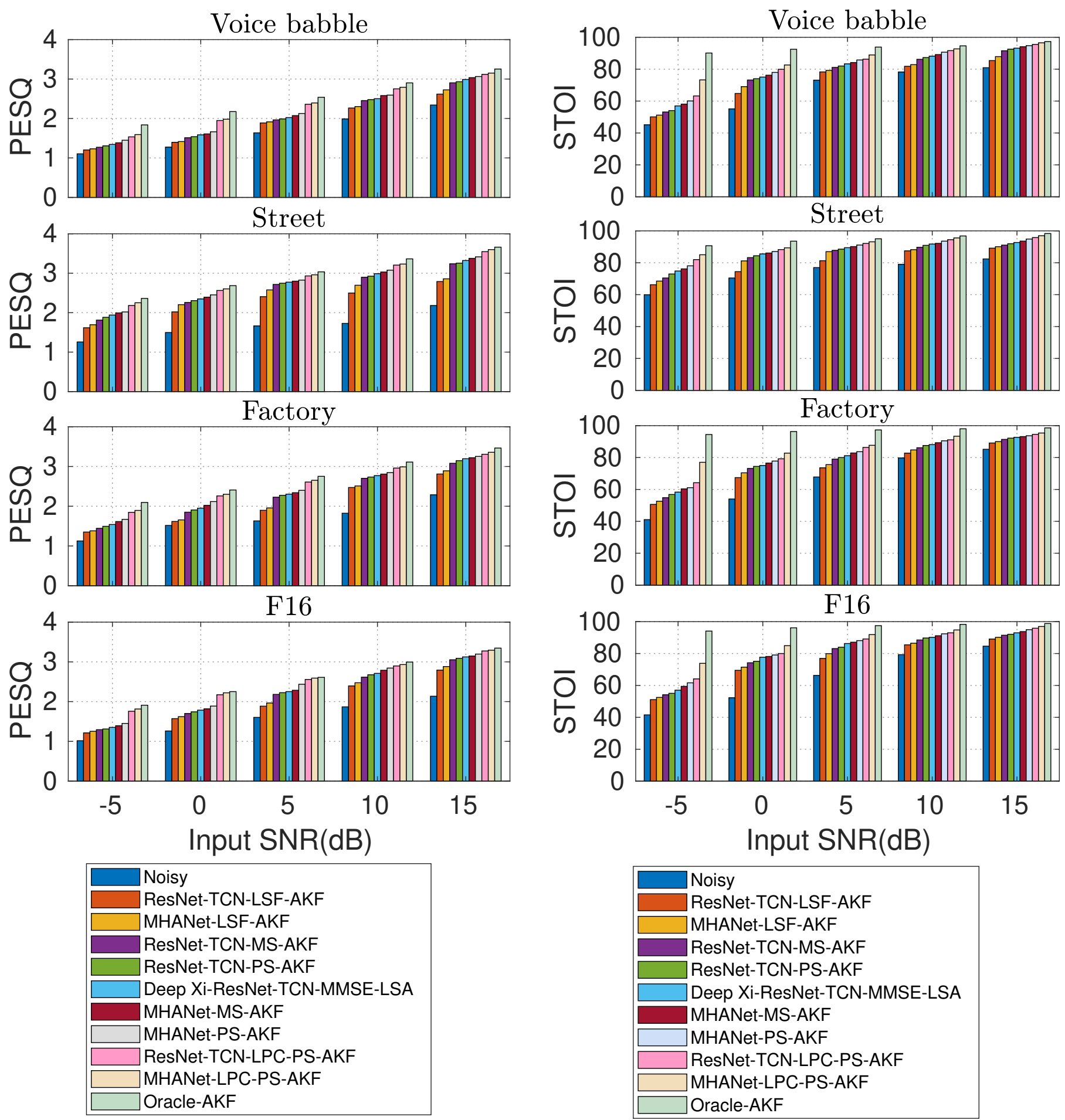

Figure 7: PESQ score for each SEA for each condition specified in Section 5.3.

\section{Conclusion}

This paper performs a comprehensive study on training targets - LSF, LPC-PS, PS, and MS using two state-ofthe-art DNNs - ResNet-TCN and MHANet for LPC estimation. This study aims to find which training target as

Figure 8: STOI score for each SEA for each condition specified in Section 5.3 .

well as DNN method estimates more accurate speech and noise LPC parameters in real-life noise conditions. The performance of the LPC estimation for each training target using ResNet-TCN and MHANet was evaluated using SD level comparisons. The accuracy of the estimated LPCs 


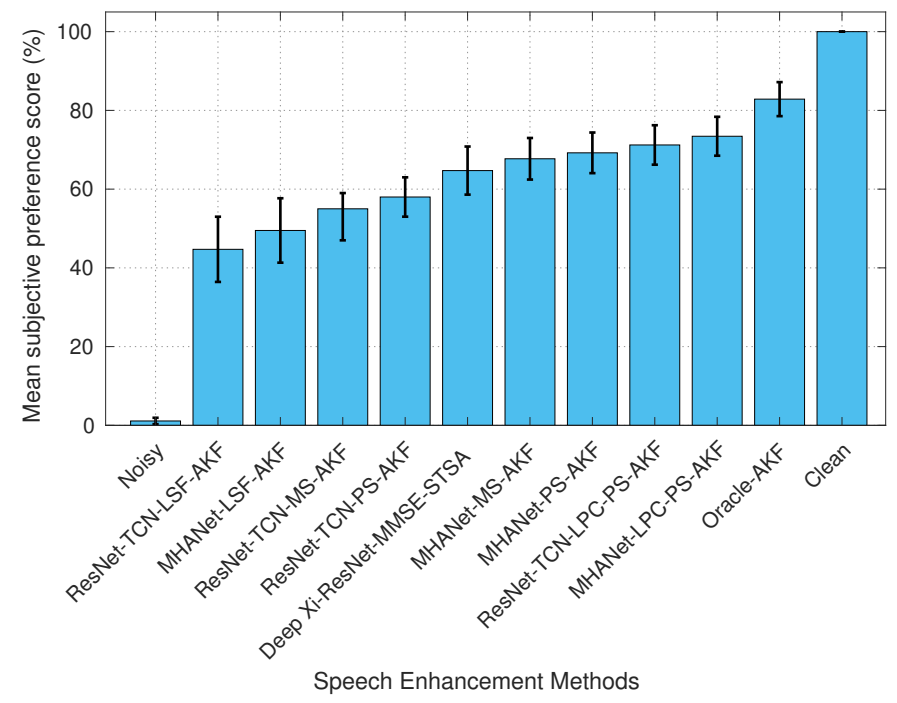

Figure 9: (Color online) The mean preference score (\%) comparison between the proposed and benchmark SEAs for the utterance sp05 corrupted with $5 \mathrm{~dB}$ voice babble noise.

was also verified in a speech enhancement context. Specifically, the $\mathrm{AKF}$ is constructed with the estimated speech and noise LPC parameters from training targets-LSF, LPC-PS, PS, and MS using ResNet-TCN and MHANet. Experiments on the NOIZEUS corpus demonstrate that the MHANet-LPC-PS produces a lower SD level in the estimated speech LPCs than other estimators in real-world non-stationary and colored noise conditions. Objective and subjective scores on the NOIZEUS corpus also indicate that the AKF constructed with the speech and noise LPC parameters derived from MHANet-LPC-PS exhibits higher quality and intelligibility in the enhanced speech than the competing methods.

\section{CRediT authorship contribution statement}

Sujan Kumar Roy: Conceptualization, Methodology, Software, Data Curation, Writing - review \& editing, Investigation, Visualization. Aaron Nocolson: Writing - review \& editing. Kuldip K. Paliwal: Supervision.

\section{Declaration of Competing Interest}

The authors declare that they have no known competing financial interests or personal relationships that could have appeared to influence the work reported in this paper.

\section{References}

Ba, L.J., Kiros, J.R., Hinton, G.E.. Layer normalization. CoRR 2016;abs/1607.06450. arXiv:1607.06450

Bai, S., Kolter, J.Z., Koltun, V.. An empirical evaluation of generic convolutional and recurrent networks for sequence modeling. ArXiv 2018;abs/1803.01271.
Dean, D.B., Sridharan, S., Vogt, R.J., Mason, M.W.. The QUT-NOISE-TIMIT corpus for the evaluation of voice activity detection algorithms. In: Proceedings Interspeech 2010. 2010. p. $3110-3113$

Ephraim, Y., Malah, D.. Speech enhancement using a minimum mean-square error log-spectral amplitude estimator. IEEE Transactions on Acoustics, Speech, and Signal Processing 1985;33(2):443-445. doi 10.1109/TASSP.1985.1164550

Garofolo, J.S., Lamel, L.F., Fisher, W.M., Fiscus, J.G., Pallett, D.S.. DARPA TIMIT acoustic-phonetic continuous speech corpus CD-ROM. NIST speech disc 1-1.1. NASA STI/Recon Technical Report N 1993;93.

George, A.E., So, S., Ghosh, R., Paliwal, K.K.. Robustness metricbased tuning of the augmented Kalman filter for the enhancement of speech corrupted with coloured noise. Speech Communication 2018;105:62-76. doi https://doi.org/10.1016/j.specom. 2018.10.002

Gibson, J.D., Koo, B., Gray, S.D.. Filtering of colored noise for speech enhancement and coding. IEEE Transactions on Signal Processing 1991;39(8):1732-1742. doi 10.1109/78.91144

Gray, A., Markel, J.. Distance measures for speech processing. IEEE Transactions on Acoustics, Speech, and Signal Processing 1976;24(5):380-391. doi 10.1109/TASSP.1976.1162849

Han, J., Pei, J., Kamber, M.. Data Mining: Concepts and Techniques. The Morgan Kaufmann Series in Data Management Systems. Elsevier Science, 2011.

He, K., Zhang, X., Ren, S., Sun, J.. Delving deep into rectifiers: Surpassing human-level performance on imagenet classification. CoRR 2015;abs/1502.01852. arXiv:1502.01852

He, K., Zhang, X., Ren, S., Sun, J.. Deep residual learning for image recognition. IEEE Conference on Computer Vision and Pattern Recognition 2016;:770-778doi 10.1109/CVPR.2016.90.

$\mathrm{Hu}$, G.. 100 nonspeech environmental sounds. The Ohio State University, Department of Computer Science and Engineering 2004;.

$\mathrm{Hu}$, Y., Loizou, P.C.. Evaluation of objective quality measures for speech enhancement. IEEE Transactions on Audio, Speech, and Language Processing 2008;16(1):229-238. doi 10.1109/TASL. 2007.911054

Itakura, F.. Line spectrum representation of linear predictor coefficients of speech signals. The Journal of the Acoustical Society of America 1975;57. doi 10.1121/1.1995189

Kamath, S., Loizou, P.. A multi-band spectral subtraction method for enhancing speech corrupted by colored noise. IEEE International Conference on Acoustics, Speech, and Signal Processing 2002;4:4160-4164. doi 10.1109/ICASSP. 2002.5745591.

Kingma, D.P., Ba, J.. Adam: A method for stochastic optimization. 2014. arXiv: 1412.6980

Loizou, P.C.. Speech Enhancement: Theory and Practice. 2nd ed. Boca Raton, FL, USA: CRC Press, Inc., 2013.

McLoughlin, I.V.. Line spectral pairs. Signal Processing 2008;88(3):448-467. doi https://doi.org/10.1016/j.sigpro. 2007.09 .003

Mermelstein, P.. Evaluation of a segmental SNR measure as an indicator of the quality of ADPCM coded speech. The Journal of the Acoustical Society of America 1979;66(6):1664-1667. doi 10. 1121/1.383638 arXiv:https://doi.org/10.1121/1.383638

Nicolson, A., Paliwal, K.K.. Deep learning for minimum meansquare error approaches to speech enhancement. Speech Communication 2019;111:44-55. doi https://doi.org/10.1016/j. specom.2019.06.002

Nicolson, A., Paliwal, K.K.. Masked multi-head self-attention for causal speech enhancement. Speech Communication 2020;125:8096. doi https://doi.org/10.1016/j.specom.2020.10.004

Paliwal, K., Basu, A.. A speech enhancement method based on Kalman filtering. IEEE International Conference on Acoustics, Speech, and Signal Processing 1987;12:177-180. doi:10.1109/ ICASSP.1987.1169756

Paliwal, K., Wójcicki, K., Schwerin, B.. Single-channel speech enhancement using spectral subtraction in the short-time modulation domain. Speech Communication 2010;52(5):450-475. doi https://doi.org/10.1016/j.specom.2010.02.004 
Panayotov, V., Chen, G., Povey, D., Khudanpur, S.. Librispeech: An ASR corpus based on public domain audio books. IEEE International Conference on Acoustics, Speech and Signal Processing 2015;:5206-5210doi 10.1109/ICASSP. 2015.7178964

Pickersgill, C., So, S., Schwerin, B.. Investigation of dnn prediction of power spectral envelopes for speech coding \& asr. 17th Speech Science and Technology Conference (SST2018), Sydney, Australia 2018 ;

Rix, A.W., Beerends, J.G., Hollier, M.P., Hekstra, A.P.. Perceptual evaluation of speech quality (PESQ)-a new method for speech quality assessment of telephone networks and codecs. IEEE International Conference on Acoustics, Speech, and Signal Processing 2001;2:749-752. doi 10.1109/ICASSP. 2001.941023

Roux, J.L., Wisdom, S., Erdogan, H., Hershey, J.R.. SDR - halfbaked or well done? In: ICASSP 2019 - 2019 IEEE International Conference on Acoustics, Speech and Signal Processing (ICASSP). 2019. p. 626-630. doi 10.1109/ICASSP. 2019.8683855

Roy, S.K., Nicolson, A., Paliwal, K.K.. A deep learning-based Kalman filter for speech enhancement. In: Proc. Interspeech 2020. 2020a. p. 2692-2696. doi 10.21437/Interspeech.2020-1551

Roy, S.K., Nicolson, A., Paliwal, K.K.. Deep learning with augmented Kalman filter for single-channel speech enhancement. In: 2020 IEEE International Symposium on Circuits and Systems (ISCAS). 2020b. p. 1-5. doi 10.1109/ISCAS45731.2020.9180820

Roy, S.K., Nicolson, A., Paliwal, K.K.. DeepLPC: A deep learning approach to augmented Kalman filter-based single-channel speech enhancement. TechRxiv 2021a;doi 10.36227/techrxiv.14384672. v1

Roy, S.K., Nicolson, A., Paliwal, K.K.. DeepLPC-MHANet: Multihead self-attention for augmented Kalman filter-based speech enhancement. TechRxiv 2021b;doi 10.36227/techrxiv.14384909. v1

Roy, S.K., Paliwal, K.K.. Causal convolutional encoder decoderbased augmented Kalman filter for speech enhancement. In: 2020 14th International Conference on Signal Processing and Communication Systems (ICSPCS). 2020a. p. 1-7. doi 10.1109/ ICSPCS50536.2020.9310011.

Roy, S.K., Paliwal, K.K.. Deep residual network-based augmented Kalman filter for speech enhancement. In: 2020 Asia-Pacific Signal and Information Processing Association Annual Summit and Conference (APSIPA ASC). 2020b. p. 667-673.

Roy, S.K., Paliwal, K.K.. Sensitivity metric-based tuning of the augmented Kalman filter for speech enhancement. 14th International Conference on Signal Processing and Communication Systems (ICSPCS) 2020 2020;doi 10.1109/ICSPCS50536. 2020.9310005

Saki, F., Kehtarnavaz, N.. Automatic switching between noise classification and speech enhancement for hearing aid devices. In: 2016 38th Annual International Conference of the IEEE Engineering in Medicine and Biology Society (EMBC). 2016. p. 736-739. doi 10.1109/EMBC. 2016.7590807.

Saki, F., Sehgal, A., Panahi, I., Kehtarnavaz, N.. Smartphonebased real-time classification of noise signals using subband features and random forest classifier. In: 2016 IEEE International Conference on Acoustics, Speech and Signal Processing (ICASSP). 2016. p. 2204-2208. doi 10.1109/ICASSP. 2016.7472068.

Snyder, D., Chen, G., Povey, D.. MUSAN: A Music, Speech, and Noise Corpus. 2015. arXiv:1510.08484 arXiv:1510.08484v1.

Srinivasan, S., Samuelsson, J., Kleijn, W.B.. Codebook driven short-term predictor parameter estimation for speech enhancement. IEEE Transactions on Audio, Speech, and Language Processing 2006;14(1):163-176. doi 10.1109/TSA.2005.854113.

Taal, C.H., Hendriks, R.C., Heusdens, R., Jensen, J.. An algorithm for intelligibility prediction of time-frequency weighted noisy speech. IEEE Transactions on Audio, Speech, and Language Processing 2011;19(7):2125-2136. doi 10.1109/TASL.2011.2114881.

Vaseghi, S.V.. Advanced Digital Signal Processing and Noise Reduction. Hoboken, NJ, USA: John Wiley Sons, Inc., 2006.

Vaswani, A., Shazeer, N., Parmar, N., Uszkoreit, J., Jones, L., Gomez, A.N., Kaiser, L.u., Polosukhin, I.. Attention is all you need. In: Guyon, I., Luxburg, U.V., Bengio, S., Wallach, H.,
Fergus, R., Vishwanathan, S., Garnett, R., editors. Advances in Neural Information Processing Systems 30. Curran Associates, Inc.; 2017. p. 5998-6008.

Veaux, C., Yamagishi, J., MacDonald, K.. CSTR VCTK corpus: English multi-speaker corpus for CSTR voice cloning toolkit. University of Edinburgh The Centre for Speech Technology Research (CSTR) 2017;

$\mathrm{Xu}$, Y., Du, J., Dai, L., Lee, C.. An experimental study on speech enhancement based on deep neural networks. IEEE Signal Processing Letters 2014;21(1):65-68. doi 10.1109/LSP . 2013.2291240

Yu, H., Ouyang, Z., Zhu, W., Champagne, B., Ji, Y.. A deep neural network based Kalman filter for time domain speech enhancement. IEEE International Symposium on Circuits and Systems 2019;:1-5doi 10.1109/ISCAS.2019.8702161.

Yu, H., Zhu, W.P., Champagne, B.. Speech enhancement using a DNN-augmented colored-noise Kalman filter. Speech Communication 2020;125:142 - 151. doi https://doi.org/10.1016/j. specom.2020.10.007

Zhang, Q., Nicolson, A., Wang, M., Paliwal, K.K., Wang, C.. DeepMMSE: A deep learning approach to MMSE-based noise power spectral density estimation. IEEE/ACM Transactions on Audio, Speech, and Language Processing 2020;28:1404-1415. doi $10.1109 /$ TASLP. 2020.2987441 